Hoai-Minh Nguyen

\title{
Further characterizations of Sobolev spaces
}

Received July 22, 2006 and in revised form December 3, 2006

Abstract. Let $\left(F_{n}\right)_{n \in \mathbb{N}}$ be a sequence of non-decreasing functions from $[0,+\infty)$ into $[0,+\infty)$. Under some suitable hypotheses on $\left(F_{n}\right)_{n \in \mathbb{N}}$, we prove that if $g \in L^{p}\left(\mathbb{R}^{N}\right), 1<p<+\infty$, satisfies

$$
\liminf _{n \rightarrow \infty} \int_{\mathbb{R}^{N}} \int_{\mathbb{R}^{N}} \frac{F_{n}(|g(x)-g(y)|)}{|x-y|^{N+p}} d x d y<+\infty,
$$

then $g \in W^{1, p}\left(\mathbb{R}^{N}\right)$ and moreover

$$
\lim _{n \rightarrow \infty} \int_{\mathbb{R}^{N}} \int_{\mathbb{R}^{N}} \frac{F_{n}(|g(x)-g(y)|)}{|x-y|^{N+p}} d x d y=K_{N, p} \int_{\mathbb{R}^{N}}|\nabla g(x)|^{p} d x,
$$

where $K_{N, p}$ is a positive constant depending only on $N$ and $p$. This extends some results in J. Bourgain and H.-M. Nguyen [A new characterization of Sobolev spaces, C. R. Math. Acad. Sci. Paris 343, 75-80 (2006)] and H.-M. Nguyen [Some new characterizations of Sobolev spaces, J. Funct. Anal. 237, 689-720 (2006)]. We also present some partial results concerning the case $p=1$ and various open problems.

Keywords. Sobolev spaces

\section{Introduction}

In [7], we established the following characterizations of Sobolev spaces:

Proposition 1 ([7], Theorem 2]). Let $1<p<+\infty$. Then

(a) There exists a constant $C_{N, p}$, depending only on $N$ and $p$, such that

$$
\int_{\mathbb{R}^{N}} \int_{\mathbb{R}^{N}(x)-g(y) \mid>\delta} \frac{\delta^{p}}{|x-y|^{N+p}} d x d y \leq C_{N, p} \int_{\mathbb{R}^{N}}|\nabla g(x)|^{p} d x, \quad \forall \delta>0, \forall g \in W^{1, p}\left(\mathbb{R}^{N}\right) .
$$

H.-M. Nguyen: Laboratoire Jacques-Louis Lions, Université Pierre et Marie Curie, 4 Place Jussieu, 75252 Paris Cedex 05, France;

e-mail: nguyen@ann.jussieu.fr, hoai-minh.nguyen@polytechnique.org

Mathematics Subject Classification (2000): Primary 46E35 
(b) If $g \in L^{p}\left(\mathbb{R}^{N}\right)$ satisfies

$$
\sup _{0<\delta<1} \int_{|g(x)-g(y)|>\delta} \int_{\mathbb{R}^{N}} \frac{\delta^{p}}{|x-y|^{N+p}} d x d y<+\infty,
$$

then $g \in W^{1, p}\left(\mathbb{R}^{N}\right)$.

(c) Moreover, for any $g \in W^{1, p}\left(\mathbb{R}^{N}\right)$,

$$
\lim _{\delta \rightarrow 0} \int_{|g(x)-g(y)|>\delta} \int_{\mathbb{R}^{N}} \frac{\delta^{p}}{|x-y|^{N+p}} d x d y=\frac{1}{p} K_{N, p} \int_{\mathbb{R}^{N}}|\nabla g(x)|^{p} d x,
$$

where $K_{N, p}$ is defined by

$$
K_{N, p}=\int_{\mathbb{S}^{N-1}}|e \cdot \sigma|^{p} d \sigma,
$$

for any $e \in \mathbb{S}^{N-1}$.

Proposition 2 ([7, Theorem 3]). Let $1<p<+\infty$. Then

(a) For every $g \in W^{1, p}\left(\mathbb{R}^{N}\right)$,

$$
\begin{array}{r}
\sup _{0<\varepsilon<1} \int_{\substack{\mathbb{R}^{N} \\
|g(x)-g(y)| \leq 1}} \int_{\mathbb{R}^{N}} \frac{\varepsilon|g(x)-g(y)|^{p+\varepsilon}}{|x-y|^{N+p}} d x d y+\int_{\mathbb{R}^{N}} \int_{\mathbb{R}^{N}} \frac{1}{|x(x)-g(y)|>1} \frac{1}{|x-y|^{N+p}} d x d y \\
\leq C_{N, p} \int_{\mathbb{R}^{N}}|\nabla g(x)|^{p} d x,
\end{array}
$$

where $C_{N, p}$ is a positive constant depending only on $N$ and $p$.

(b) If $g \in L^{p}\left(\mathbb{R}^{N}\right)$ satisfies

$$
\sup _{0<\varepsilon<1} \int_{\substack{\mathbb{R}^{N} \\|g(x)-g(y)| \leq 1}} \int_{\mathbb{R}^{N}} \frac{\varepsilon|g(x)-g(y)|^{p+\varepsilon}}{|x-y|^{N+p}} d x d y+\int_{\mathbb{R}^{N}} \int_{\mathbb{R}^{N}} \frac{1}{|g(x)-g(y)|>1} \frac{1}{|x-y|^{N+p}} d x d y<+\infty,
$$

then $g \in W^{1, p}\left(\mathbb{R}^{N}\right)$.

(c) Moreover, for any $g \in W^{1, p}\left(\mathbb{R}^{N}\right)$,

$$
\lim _{\varepsilon \rightarrow 0} \int_{|g(x)-g(y)| \leq 1} \int_{\mathbb{R}^{N}} \frac{\varepsilon|g(x)-g(y)|^{p+\varepsilon}}{|x-y|^{N+p}} d x d y=K_{N, p} \int_{\mathbb{R}^{N}}|\nabla g(x)|^{p} d x,
$$

where $K_{N, p}$ is defined by (1.1).

A sharper version of assertion (b) of Proposition 1 was established by J. Bourgain and H.-M. Nguyen in [2]: 
Proposition 3 ([2, Theorem 1]). Let $g \in L^{p}\left(\mathbb{R}^{N}\right), 1<p<+\infty$, be such that

$$
\sup _{n \in \mathbb{N}} \int_{|g(x)-g(y)|>\delta_{n}} \int_{\mathbb{R}^{N}} \frac{\delta_{n}^{p}}{|x-y|^{N+p}} d x d y<+\infty
$$

for some sequence $\left(\delta_{n}\right)_{n \in \mathbb{N}}$ of positive numbers with $\lim _{n \rightarrow \infty} \delta_{n}=0$. Then $g \in W^{1, p}\left(\mathbb{R}^{N}\right)$.

When $p=1$, we have

Proposition 4. Let $g \in L^{1}\left(\mathbb{R}^{N}\right)$ be such that

$$
\sup _{n \in \mathbb{N}} \int_{|g(x)-g(y)|>\delta_{n}} \int_{\mathbb{R}^{N}} \frac{\delta_{n}}{|x-y|^{N+1}} d x d y<+\infty
$$

for some sequence $\left(\delta_{n}\right)_{n \in \mathbb{N}}$ of positive numbers with $\lim _{n \rightarrow \infty} \delta_{n}=0$. Then $g \in B V\left(\mathbb{R}^{N}\right)$; moreover, there exists a constant $c_{N}$, depending only on $N$, such that

$$
\liminf _{n \rightarrow \infty} \int_{|g(x)-g(y)|>\delta_{n}} \int_{\mathbb{R}^{N}} \frac{\delta_{n}}{|x-y|^{N+1}} d x d y \geq c_{N} \int_{\mathbb{R}^{N}}|\nabla g| d x
$$

Remark 1. Proposition 4 is not stated explicitly in 2, but its proof is implicit there (see the proof of [2, Theorem 1]).

The proof of Proposition 3 is much more involved than the one of Propositions 1 and 2 (see [2]).

In this paper, we generalize Propositions 13 as follows:

Theorem 1. Let $1<p<+\infty$ and $\left(F_{n}\right)_{n \in \mathbb{N}}$ be a sequence of functions from $[0,+\infty)$ into $[0,+\infty)$ such that

(i) $F_{n}(t)$ is a non-decreasing function with respect to $t$ on $[0,+\infty)$, for all $n \in \mathbb{N}$.

(ii) $\int_{0}^{1} F_{n}(t) t^{-(p+1)} d t=1$ for all $n \in \mathbb{N}$.

(iii) $F_{n}(t)$ converges uniformly to 0 on every compact subset of $(0,+\infty)$ as $n$ goes to infinity.

Then

(a) If $g \in W^{1, p}\left(\mathbb{R}^{N}\right)$, then for every $n \in \mathbb{N}$,

$$
\int_{\mathbb{R}^{N}} \int_{\mathbb{R}^{N}} \frac{F_{n}(|g(x)-g(y)|)}{|x-y|^{N+p}} d x d y \leq C_{N, p} \int_{0}^{\infty} F_{n}(t) t^{-(p+1)} d t \int_{\mathbb{R}^{N}}|\nabla g(x)|^{p} d x,
$$

where $C_{N, p}$ is a positive constant depending only on $N$ and $p$. 
(b) If $g \in L^{p}\left(\mathbb{R}^{N}\right)$ satisfies

$$
\liminf _{n \rightarrow \infty} \int_{\mathbb{R}^{N}} \int_{\mathbb{R}^{N}} \frac{F_{n}(|g(x)-g(y)|)}{|x-y|^{N+p}} d x d y<+\infty,
$$

then $g \in W^{1, p}\left(\mathbb{R}^{N}\right)$ and

$$
\liminf _{n \rightarrow \infty} \int_{\mathbb{R}^{N}} \int_{\mathbb{R}^{N}} \frac{F_{n}(|g(x)-g(y)|)}{|x-y|^{N+p}} d x d y \geq K_{N, p} \int_{\mathbb{R}^{N}}|\nabla g(x)|^{p} d x .
$$

(c) Moreover, if

$$
\limsup _{n \rightarrow \infty} \int_{0}^{\infty} F_{n}(t) t^{-(p+1)} d t<+\infty
$$

then

$\lim _{n \rightarrow \infty} \int_{\mathbb{R}^{N}} \int_{\mathbb{R}^{N}} \frac{F_{n}(|g(x)-g(y)|)}{|x-y|^{N+p}} d x d y=K_{N, p} \int_{\mathbb{R}^{N}}|\nabla g(x)|^{p} d x, \quad \forall g \in W^{1, p}\left(\mathbb{R}^{N}\right)$.

Here $K_{N, p}$ is defined by 1.1 .

Remark 2. Many ideas used in the proof of Theorems 1 and 2 are borrowed from the method of J. Bourgain and H.-M. Nguyen in [2].

Remark 3. Propositions 1 and 3 follow from Theorem 1 by choosing

$$
F_{n}(t)= \begin{cases}0 & \text { if } 0 \leq t \leq \delta_{n} \\ \frac{p \delta_{n}^{p}}{1-\delta_{n}^{p}} & \text { otherwise. }\end{cases}
$$

To deduce Proposition 2 we choose

$$
F_{n}(t)= \begin{cases}\varepsilon_{n} t^{p+\varepsilon_{n}} & \text { if } 0 \leq t \leq 1, \\ \varepsilon_{n} & \text { otherwise. }\end{cases}
$$

Remark 4. We now make some comments about hypotheses (i)-(iii) on the sequence $\left(F_{n}\right)$. The conclusion of Theorem 1 may fail if we do not assume (i). For example, let

$$
F_{n}(t)=\left\{\begin{array}{ll}
n t^{p+1} & \text { if } 0 \leq t<1 / n, \\
0 & \text { otherwise }
\end{array} \quad g(x)= \begin{cases}1 & \text { if }|x| \leq 1, \\
0 & \text { otherwise }\end{cases}\right.
$$

Clearly,

$$
\int_{\mathbb{R}^{N}} \int_{\mathbb{R}^{N}} \frac{F_{n}(|g(x)-g(y)|)}{|x-y|^{N+p}} d x d y=0, \quad \forall n \geq 1, \forall p>1,
$$

but $g \notin W^{1, p}\left(\mathbb{R}^{N}\right)$ for all $p>1$. 
Condition (ii) is a normalization condition. Indeed, if we assume

$$
\lim _{n \rightarrow \infty} \int_{0}^{1} F_{n}(t) t^{-(p+1)} d t=+\infty
$$

then $g$ is a constant function (see Corollary 1).

Condition (iii) is also important. Indeed, the sequence $F_{n}(t)=t^{p+1}$, for all $n \geq 1$, satisfies conditions (i) and (ii). However, condition (1.6) is equivalent to $g \in W^{p /(p+1), p+1}\left(\mathbb{R}^{N}\right)$.

The analogue of assertion (b) in Theorem 1 for $p=1$ is the following

Theorem 2. Let $\left(F_{n}\right)_{n \in \mathbb{N}}$ be a sequence of functions from $[0,+\infty)$ into $[0,+\infty)$ satisfying (i), (ii) with $p=1$ and (iii). Assume that $g \in L^{1}\left(\mathbb{R}^{N}\right)$ and $g$ satisfies (1.6) with $p=1$. Then $g \in B V\left(\mathbb{R}^{N}\right)$. Moreover, there exists a constant $c_{N}$, depending only on $N$, such that

$$
\liminf _{n \rightarrow \infty} \int_{\mathbb{R}^{N}} \int_{\mathbb{R}^{N}} \frac{F_{n}(|g(x)-g(y)|)}{|x-y|^{N+1}} d x d y \geq c_{N} \int_{\mathbb{R}^{N}}|\nabla g| d x .
$$

Comparing with (1.7), we have

Question 1. Can one replace $c_{N}$ by $K_{N, 1}$ in 1.11 ?

The reader can find further questions in Section 4.

Remark 5. Proposition 4 follows from Theorem 2 by choosing $F_{n}$ as in (1.10) with $p=1$.

For what concerns the analogues of assertions (a) and (c), A. Ponce has constructed a function $g \in W^{1,1}(\mathbb{R})$ such that

$$
\lim _{\delta \rightarrow 0} \int_{|g(x)-g(y)|>\delta} \int_{\mathbb{R}} \frac{\delta}{|x-y|^{2}} d x d y=+\infty .
$$

Hence the analogues of these assertions for $p=1$ do not hold (see [7]).

The proof of assertion (a) in Theorem 1 is similar to one in [7]; it is based on maximal functions. We present two methods of proof of assertion (b) in Theorem 1 . The first one, is based on Proposition 3 The second one which relies heavily on Lemma 2 below, is more complicated but is interesting in its own right. For what concerns Theorem 2, we are able to apply the first method, but not the second due to lack of an analogue of Lemma 2 for $p=1$. Lemma 2 is closely related to Proposition 3, its proof uses many ideas of J. Bourgain and H.-M. Nguyen from [2]. The proof of assertion (c) in Theorem 1 is also much more delicate than the one of assertion (c) in Propositions 1 and 2

The paper is organized as follows. In Section 2 we will prove Theorems 1 and 2. In Section 3 we present another proof of assertion (b) in Theorem 1 Finally, in Section 4 we will discuss problems related to $\Gamma$-convergence. 


\section{Proofs of Theorems 1 and 2}

\subsection{Proof of assertion (a) in Theorem 1}

Using the change of variables formula and Fubini's theorem, one gets

$$
\begin{aligned}
\int_{\mathbb{R}^{N}} \int_{\mathbb{R}^{N}} \frac{F_{n}(|g(x)-g(y)|)}{|x-y|^{N+p}} & d x d y \\
= & \int_{\mathbb{S}^{N-1}} \int_{\mathbb{R}^{N}} \int_{0}^{\infty} \frac{F_{n}(|g(x+h \sigma)-g(x)|)}{h^{p+1}} d h d x d \sigma .
\end{aligned}
$$

Consequently, to prove (1.5), it suffices to show that

$$
\int_{\mathbb{R}^{N}} \int_{0}^{\infty} \frac{F_{n}(|g(x+h \sigma)-g(x)|)}{h^{p+1}} d h d x \leq C_{p} \int_{0}^{\infty} F_{n}(t) t^{-(p+1)} d t \int_{\mathbb{R}^{N}}|\nabla g(x)|^{p} d x
$$

for all $n \in \mathbb{N}$, where $C_{p}$ is a positive constant depending only on $p$.

Without loss of generality we may assume that $\sigma=e_{N}$. Since $g \in W^{1, p}\left(\mathbb{R}^{N}\right)$, $g\left(x^{\prime}, \cdot\right) \in W^{1, p}(\mathbb{R})$ for almost every $x^{\prime}=\left(x_{1}, \ldots, x_{N-1}\right) \in \mathbb{R}^{N-1}$.

Fix $x^{\prime} \in \mathbb{R}^{N-1}$ such that $g\left(x^{\prime}, \cdot\right) \in W^{1, p}(\mathbb{R})$. Then

$$
\left|g\left(x+h e_{N}\right)-g(x)\right| \leq h \int_{x_{N}}^{x_{N}+h}\left|\frac{\partial g}{\partial x_{N}}\left(x^{\prime}, s\right)\right| d s \leq h M_{N}\left(\frac{\partial g}{\partial x_{N}}\right)(x)
$$

for almost every $\left(x_{N}, h\right) \in \mathbb{R} \times(0,+\infty)$, where $M_{N}(f)$ denotes the maximal function of $f$ with respect to the variable $x_{N}$ in the positive direction, i.e.,

$$
M_{N}(f)\left(x^{\prime}, x_{N}\right)=\sup _{h>0} \int_{x_{N}}^{x_{N}+h}\left|f\left(x^{\prime}, s\right)\right| d s .
$$

Hence, since $F_{n}(t)$ is a non-decreasing function with respect to $t$,

$$
F_{n}\left(\left|g\left(x+h e_{N}\right)-g(x)\right|\right) \leq F_{n}\left(h M_{N}\left(\frac{\partial g}{\partial x_{N}}\right)(x)\right) \quad \text { for a.e. }\left(x_{N}, h\right) \in \mathbb{R} \times(0,+\infty),
$$

which shows that

$$
\int_{\mathbb{R}^{N}} \int_{0}^{\infty} \frac{F_{n}\left(\left|g\left(x+h e_{N}\right)-g(x)\right|\right)}{h^{p+1}} d h d x \leq \int_{\mathbb{R}^{N}} \int_{0}^{\infty} \frac{F_{n}\left(h M_{N}\left(\partial g / \partial x_{N}\right)(x)\right)}{h^{p+1}} d h d x .
$$

A direct computation yields

$$
\begin{aligned}
& \int_{\mathbb{R}^{N}} \int_{0}^{\infty} \frac{F_{n}\left(\mid g\left(x+h e_{N}\right)-\right.}{h^{p+1}} d h d x \\
& \leq \int_{\mathbb{R}^{N}}\left|M_{N}\left(\frac{\partial g}{\partial x_{N}}\right)(x)\right|^{p} d x \int_{0}^{\infty} F_{n}(h) h^{-(p+1)} d h .
\end{aligned}
$$


On the other hand, using the theory of maximal functions (see, e.g., [9, Chapter 1]), one finds

$$
\int_{\mathbb{R}^{N-1}} \int_{\mathbb{R}}\left|M_{N}\left(\frac{\partial g}{\partial x_{N}}\right)(x)\right|^{p} d x_{N} d x^{\prime} \leq C_{p} \int_{\mathbb{R}^{N-1}} \int_{\mathbb{R}^{\mathbb{N}}}\left|\frac{\partial g}{\partial x_{N}}(x)\right|^{p} d x_{N} d x^{\prime} .
$$

Consequently,

$$
\int_{\mathbb{R}^{N}}\left|M_{N}\left(\frac{\partial g}{\partial x_{N}}\right)(x)\right|^{p} d x \leq C_{p} \int_{\mathbb{R}^{N}}|\nabla g(x)|^{p} d x .
$$

Therefore 2.1 is proved and 1.5 follows.

\subsection{Proof of assertion (c) in Theorem 1}

The following lemma is useful in the proof of assertion (c) in Theorem 1 .

Lemma 1. Assume that $g \in W^{1, p}(\mathbb{R})$ and $\left(F_{n}\right)_{n \in \mathbb{N}}$ satisfies hypotheses (i)-(iii) of Theorem 1 and 1.8 . Then

$$
\lim _{n \rightarrow \mathbb{N}} \int_{\mathbb{R}} \int_{0}^{\infty} \frac{F_{n}(|g(t+h)-g(t)|)}{h^{p+1}} d h d t=\int_{\mathbb{R}}\left|g^{\prime}(t)\right|^{p} d t .
$$

Proof. Since $g \in W^{1, p}(\mathbb{R})$,

$|g(t+h)-g(t)| \leq h \int_{t}^{t+h}\left|g^{\prime}(s)\right| d s \leq h\left|M_{+}\left(g^{\prime}\right)(t)\right| \quad$ for a.e. $(t, h) \in \mathbb{R} \times(0,+\infty)$,

where $M_{+}\left(g^{\prime}\right)$ denotes the maximal function of $g^{\prime}$ in the positive direction, i.e.,

$$
M_{+}\left(g^{\prime}\right)(t)=\sup _{h>0} \int_{t}^{t+h}\left|g^{\prime}(s)\right| d s .
$$

Hence, since $F_{n}$ is a non-decreasing function, it follows that, for all measurable sets $A \subset \mathbb{R}$

$$
\begin{aligned}
& \int_{A} \int_{0}^{\infty} \frac{F_{n}(|g(t+h)-g(t)|)}{h^{p+1}} d h d t \\
& \leq \sup _{n \in \mathbb{N}} \int_{0}^{\infty} F_{n}(h) h^{-(p+1)} d h \int_{A}\left|M_{+}\left(g^{\prime}\right)(t)\right|^{p} d t .
\end{aligned}
$$

On the other hand, since $g \in W^{1, p}(\mathbb{R})$ and $1<p<+\infty$, applying the theory of maximal functions (see [9, Chapter 1]), one gets $M_{+}\left(g^{\prime}\right) \in L^{p}(\mathbb{R})$ and

$$
\int_{\mathbb{R}}\left|M_{+}\left(g^{\prime}\right)(x)\right|^{p} d x \leq C \int_{\mathbb{R}}\left|g^{\prime}(x)\right|^{p} d x .
$$


Hereafter in this proof $C$ will denote a positive constant depending only on $p$. Thus it follows from 2.4 that for each $\varepsilon \in(0,1)$, there exists a positive constant $k=k(\varepsilon) \geq 1$ such that

$$
\int_{\mathbb{R} \backslash B} \int_{0}^{\infty} \frac{F_{n}(|g(t+h)-g(t)|)}{h^{p+1}} d h d t+\int_{\mathbb{R} \backslash B}\left|g^{\prime}(t)\right|^{p} d t \leq \varepsilon / 2,
$$

where

$$
B:=\left\{t \in[-k, k] ;\left|g^{\prime}(t)\right| \leq k\right\}
$$

Set, for each $\tau>0$,

$$
A_{\tau}=\left\{t \in B ; q(t) \leq \frac{|g(t+h)-g(t)|}{h} \leq\left|g^{\prime}(t)\right|+\gamma \text { for a.e. } h \in[0, \tau]\right\},
$$

where $\gamma=\varepsilon / k^{p}$ and $q(t)$ is defined as follows:

$$
q(t)= \begin{cases}\left|g^{\prime}(t)\right|-\gamma & \text { if }\left|g^{\prime}(t)\right| \geq \gamma \\ 0 & \text { otherwise }\end{cases}
$$

Since $g \in W^{1, p}(\mathbb{R})$, it follows from (2.4) and (2.5) that one can choose $\tau$ sufficiently small such that

$$
\int_{B \backslash A_{\tau}} \int_{0}^{\infty} \frac{F_{n}(|g(t+h)-g(t)|)}{h^{p+1}} d h d t+\int_{B \backslash A_{\tau}}\left|g^{\prime}(t)\right|^{p} d t \leq \varepsilon / 2 .
$$

On the other hand, since $F_{n}$ is a non-decreasing function,

$$
\int_{A_{\tau}} \int_{0}^{\tau} \frac{F_{n}(|g(t+h)-g(t)|)}{h^{p+1}} d h d t \leq \int_{A_{\tau}} \int_{0}^{\tau} \frac{F_{n}\left(\left(\left|g^{\prime}(t)\right|+\gamma\right) h\right)}{h^{p+1}} d h d t .
$$

A direct computation yields

$\int_{A_{\tau}} \int_{0}^{\tau} \frac{F_{n}\left(\left(\left|g^{\prime}(t)\right|+\gamma\right) h\right)}{h^{p+1}} d h d t=\int_{A_{\tau}}\left(\left|g^{\prime}(t)\right|+\gamma\right)^{p} \int_{0}^{\left(\left|g^{\prime}(t)\right|+\gamma\right) \tau} F_{n}(s) s^{-(p+1)} d s d t$.

Moreover, since $\int_{0}^{1} F_{n}(t) t^{-(p+1)} d t=1$ and $F_{n}(t)$ converges uniformly to 0 on every compact subset of $(0,+\infty)$ as $n$ goes to infinity,

$$
\lim _{n \rightarrow \infty} \int_{A_{\tau}}\left(\left|g^{\prime}(t)\right|+\gamma\right)^{p} \int_{0}^{\left(\left|g^{\prime}(t)\right|+\gamma\right) \tau} F_{n}(s) s^{-(p+1)} d s d t=\int_{A_{\tau}}\left(\left|g^{\prime}(t)\right|+\gamma\right)^{p} d t .
$$

Therefore,

$$
\limsup _{n \rightarrow \infty} \int_{A_{\tau}} \int_{0}^{\tau} \frac{F_{n}(|g(t+h)-g(t)|)}{h^{p+1}} d h d t \leq \int_{A_{\tau}}\left(\left|g^{\prime}(t)\right|+\gamma\right)^{p} d t .
$$


Furthermore,

$$
\begin{aligned}
\int_{\mathbb{R}}\left(\int_{0}^{\tau} \frac{F_{n}(|g(t+h)-g(t)|)}{h^{p+1}} d h-\left|g^{\prime}(t)\right|^{p}\right) d t \\
=\int_{\mathbb{R} \backslash B}\left(\int_{0}^{\tau} \frac{F_{n}(|g(t+h)-g(t)|)}{h^{p+1}} d h-\left|g^{\prime}(t)\right|^{p}\right) d t \\
+\int_{B \backslash A_{\tau}}\left(\int_{0}^{\tau} \frac{F_{n}(|g(t+h)-g(t)|)}{h^{p+1}} d h-\left|g^{\prime}(t)\right|^{p}\right) d t \\
+\int_{A_{\tau}}\left(\int_{0}^{\tau} \frac{F_{n}(|g(t+h)-g(t)|)}{h^{p+1}} d h-\left|g^{\prime}(t)\right|^{p}\right) d t .
\end{aligned}
$$

Thus combining (2.6), (2.8), and (2.9) yields

$$
\begin{aligned}
\int_{\mathbb{R}} \int_{0}^{\tau} \frac{F_{n}(|g(t+h)-g(t)|)}{h^{p+1}} d h d t-\int_{\mathbb{R}}\left|g^{\prime}(t)\right|^{p} d t \\
\leq 3 \varepsilon+\int_{A_{\tau}}\left(\left(\left|g^{\prime}(t)+\gamma\right|\right)^{p}-\left|g^{\prime}(t)\right|^{p}\right) d t,
\end{aligned}
$$

when $n \geq n_{\varepsilon}$.

Since $(a+\gamma)^{p} \leq a^{p}(1+C \gamma / a)$ if $\gamma \leq a$ and $(a+\gamma)^{p} \leq C \gamma$ if $a \leq \gamma \leq 1$, one has

$$
\int_{A_{\tau}}\left(\left(\left|g^{\prime}(t)+\gamma\right|\right)^{p}-\left|g^{\prime}(t)\right|^{p}\right) d t \leq C \gamma k^{p}=C \varepsilon .
$$

Here we use the fact that $A_{\tau} \subset B$ and the choice of $\gamma=\varepsilon / k^{p}$. Hence it follows from 2.10 that

$$
\int_{\mathbb{R}} \int_{0}^{\tau} \frac{F_{n}(|g(t+h)-g(t)|)}{h^{p+1}} d h d t-\int_{\mathbb{R}}\left|g^{\prime}(t)\right|^{p} d t \leq C \varepsilon, \quad \forall n \geq n_{\varepsilon} .
$$

Similarly,

$$
\begin{aligned}
\int_{\mathbb{R}} \int_{0}^{\tau} \frac{F_{n}(|g(t+h)-g(t)|)}{h^{p+1}} d h d t- & \int_{\mathbb{R}}\left|g^{\prime}(t)\right|^{p} d t \\
& \geq-3 \varepsilon+\int_{A_{\tau}}\left(q^{p}(t)-\left|g^{\prime}(t)\right|^{p}\right) d t
\end{aligned}
$$

for all $n \geq n_{\varepsilon}$. Recall here that the function $q$ is defined by 2.7p. Since $(a-\gamma)^{p}+$ $C \gamma a^{p-1} \geq a^{p}$ for all $a \geq \gamma>0$ and $A_{\tau} \subset B$, one deduces that

$$
\int_{A_{\tau}}\left(q^{p}(t)-\left|g^{\prime}(t)\right|^{p}\right) d t \geq-C \gamma k^{p}=-C \varepsilon
$$

Thus, from 2.12,

$$
\int_{\mathbb{R}} \int_{0}^{\tau} \frac{F_{n}(|g(t+h)-g(t)|)}{h^{p+1}} d h d t-\int_{\mathbb{R}}\left|g^{\prime}(t)\right|^{p} d t \geq-C \varepsilon, \quad \forall n \geq n_{\varepsilon} .
$$


Combining 2.11) and 2.13) yields

$$
\left.\left|\int_{\mathbb{R}} \int_{0}^{\tau} \frac{F_{n}(|g(t+h)-g(t)|)}{h^{p+1}} d h d t-\int_{\mathbb{R}}\right| g^{\prime}(t)\right|^{p} d t \mid \leq C \varepsilon, \quad \forall n \geq n_{\varepsilon} .
$$

On the other hand, since $g \in W^{1, p}(\mathbb{R})$, it follows that $g \in L_{\text {loc }}^{\infty}(\mathbb{R})$. Thus since $F_{n}(t)$ converges uniformly to 0 on every compact subset of $(0,+\infty)$, applying Lebesgue's dominated convergence theorem, one obtains

$$
\lim _{n \rightarrow \infty} \int_{|t| \leq k} \int_{\tau}^{m} \frac{F_{n}(|g(t+h)-g(t)|)}{h^{p+1}} d h d t=0, \quad \forall m>0 .
$$

Moreover, since $F_{n}$ is a non-decreasing function, it follows that

$$
\begin{aligned}
\int_{|t| \leq k} \int_{m}^{\infty} \frac{F_{n}(|g(t+h)-g(t)|)}{h^{p+1}} d h d t & \leq 2 k \int_{m}^{\infty} \frac{F_{n}\left(\left\|g^{\prime}\right\|_{L^{p}} h^{(p-1) / p}\right)}{h^{p+1}} d h \\
& \leq \frac{2 k}{m}\left\|g^{\prime}\right\|_{L^{p}}^{p} \int_{0}^{\infty} F_{n}(t) t^{-(p+1)} d t .
\end{aligned}
$$

Hence using [1.8], one gets

$$
\lim _{n \rightarrow \infty} \int_{|t| \leq k} \int_{\tau}^{\infty} \frac{F_{n}(|g(t+h)-g(t)|)}{h^{p+1}} d h d t=0 .
$$

Therefore the conclusion of Lemma 1 follows from 2.6, 2.14), and 2.15).

Proof of assertion (c). We claim that

$$
\lim _{n \rightarrow \infty} \int_{\mathbb{R}^{N}} \int_{0}^{\infty} \frac{F_{n}(|g(x+h \sigma)-g(x)|)}{h^{p+1}} d h d x=\int_{\mathbb{R}^{N}}|\nabla g(x) \cdot \sigma|^{p} d x .
$$

Without loss of generality, one may assume that $\sigma=e_{N}$. Take $x^{\prime} \in \mathbb{R}^{N-1}$ such that $g\left(x^{\prime}, \cdot\right) \in W^{1, p}(\mathbb{R})$. As in 2.4, one gets

$$
\begin{aligned}
\int_{\mathbb{R}} \int_{0}^{\infty} \frac{F_{n}\left(\left|g\left(x^{\prime}, x_{N}+h\right)-g\left(x^{\prime}, x_{N}\right)\right|\right)}{h^{p+1}} d h d x_{N} \\
\leq \int_{0}^{\infty} F_{n}(t) t^{-(p+1)} d t \int_{\mathbb{R}}\left|M_{N}\left(\partial g / \partial x_{N}\right)\left(x^{\prime}, x_{N}\right)\right|^{p} d x_{N} .
\end{aligned}
$$

Here $M_{N}$ is defined by 2.2. On the other hand, by Lemma1.

$$
\lim _{n \rightarrow \infty} \int_{\mathbb{R}} \int_{0}^{\infty} \frac{F_{n}\left(\left|g\left(x^{\prime}, x_{N}+h\right)-g\left(x^{\prime}, x_{N}\right)\right|\right)}{h^{p+1}} d h d x_{N}=\int_{\mathbb{R}}\left|\frac{\partial g}{\partial x_{N}}\left(x^{\prime}, x_{N}\right)\right|^{p} d x_{N} .
$$

Thus, applying Lebesgue's dominated convergence theorem, one obtains

$$
\lim _{n \rightarrow \infty} \int_{\mathbb{R}^{N}} \int_{0}^{\infty} \frac{F_{n}\left(\left|g\left(x+h e_{N}\right)-g(x)\right|\right)}{h^{p+1}} d h d x=\int_{\mathbb{R}^{N}}\left|\nabla g(x) \cdot e_{N}\right|^{p} d x .
$$

Therefore the conclusion of assertion (c) in Theorem 1 follows from 2.1, 2.16 and Lebesgue's dominated convergence theorem. 


\subsection{Proof of assertion (b) in Theorem 1}

Without loss of generality, we may assume that

$$
M:=1+\sup _{n \in \mathbb{N}} \int_{\mathbb{R}^{N}} \int_{\mathbb{R}^{N}} \frac{F_{n}(|g(x)-g(y)|)}{|x-y|^{p+N}} d x d y<+\infty .
$$

Thus since $F_{n}(t)$ is a non-decreasing function with respect to $t$,

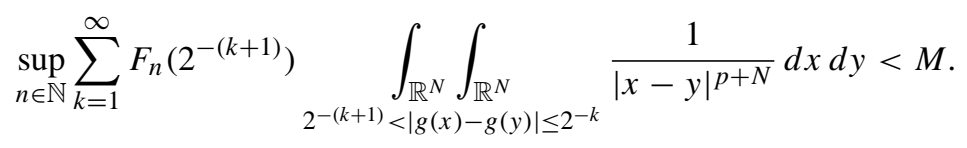

On the other hand, by (i)-(iii) it follows that for each $s>0$ there exists $n$ such that $F_{n}(s)>0$. Thus since $F_{n}$ is a non-decreasing function,

$$
\int_{\mathbb{R}^{N}} \int_{\mathbb{R}^{N}} \frac{1}{|x(x)-g(y)|>s} \frac{1}{\mid x-N} d x d y<+\infty, \quad \forall s>0 .
$$

Hence

$$
\begin{aligned}
& \sum_{k=1}^{\infty} F_{n}\left(2^{-(k+1)}\right) \int_{\substack{\mathbb{R}^{N} \\
2^{-(k+1)}<|g(x)-g(y)| \leq 2^{-k}}} \int_{\mathbb{R}^{N}} \frac{1}{|x-y|^{p+N}} d x d y \\
& =\sum_{k=1}^{\infty} F_{n}\left(2^{-(k+1)}\right) \\
& \times\left(\int_{\mathbb{R}^{N}} \int_{\mathbb{R}^{N}} \frac{1}{|x-y|^{p+N}} d x d y-\int_{\mathbb{R}^{N}} \int_{\mathbb{R}^{N}} \frac{1}{|x(x)-g(y)|>2^{-k}} \frac{1}{|x-y|^{p+N}} d x d y\right) .
\end{aligned}
$$

However, the right hand side above equals

$$
\begin{aligned}
& \sum_{k=1}^{\infty} 2^{p(k+1)} F_{n}\left(2^{-(k+1)}\right) \int_{\substack{\mathbb{R}^{N} \\
|g(x)-g(y)|>2^{-(k+1)}}} \int_{\mathbb{R}^{N}} \frac{2^{-(k+1) p}}{|x-y|^{p+N}} d x d y \\
& -\sum_{k=1}^{\infty} 2^{p k} F_{n}\left(2^{-(k+1)}\right) \int_{\substack{\mathbb{R}^{N} \\
|g(x)-g(y)|>2^{-k}}} \frac{2^{-k p}}{|x-y|^{p+N}} d x d y \\
& =\sum_{k=2}^{\infty} 2^{p k}\left(F_{n}\left(2^{-k}\right)-F_{n}\left(2^{-(k+1)}\right)\right) \int_{|g(x)-g(y)|>2^{-k}} \int_{\mathbb{R}^{N}} \frac{2^{-k p}}{|x-y|^{p+N}} d x d y \\
& -F_{n}(1 / 4) \int_{\mathbb{R}^{N}} \int_{\mathbb{R}^{N}} \frac{1}{|x-y|^{p+N}} d x d y
\end{aligned}
$$


and, from hypothesis (iii) on $F_{n}$,

$$
\lim _{n \rightarrow \infty} F_{n}(1 / 4)=0
$$

Thus it follows from 2.18 that

$$
\limsup _{n \rightarrow \infty} \sum_{k=2}^{\infty} 2^{p k}\left(F_{n}\left(2^{-k}\right)-F_{n}\left(2^{-(k+1)}\right)\right) \int_{\substack{\mathbb{R}^{N} \\|g(x)-g(y)|>2^{-k}}} \int_{\mathbb{R}^{N}} \frac{2^{-k p}}{|x-y|^{p+N}} d x d y \leq M .
$$

We claim that

$$
\liminf _{k \rightarrow \infty} \int_{\mathbb{R}^{N}} \int_{\mathbb{R}^{N}} \frac{2^{-k p}}{|x-y|^{p+N}} d x d y<+\infty .
$$

We prove 2.20) by contradiction. Suppose it does not hold. Then there exists $k_{M} \in \mathbb{N}$ such that for all $k \geq k_{M}$,

$$
\int_{\mathbb{R}^{N}} \int_{\mathbb{R}^{N}} \frac{2^{-k p}}{|x-y|^{p+N}} d x d y>2^{p+2} M
$$

Hence it follows from 2.19, that

$$
\limsup _{n \rightarrow \infty} 2^{p+2} M \sum_{k=k_{M}}^{\infty} 2^{p k}\left(F_{n}\left(2^{-k}\right)-F_{n}\left(2^{-(k+1)}\right)\right) \leq M,
$$

which shows that

$$
\limsup _{n \rightarrow \infty} \sum_{k=k_{M}}^{\infty} 2^{p k}\left(F_{n}\left(2^{-k}\right)-F_{n}\left(2^{-(k+1)}\right)\right) \leq 2^{-(p+2)} .
$$

On the other hand, since $p \geq 1$,

$$
\begin{aligned}
\sum_{k=k_{M}}^{\infty} 2^{p k}\left(F_{n}\left(2^{-k}\right)-F_{n}\left(2^{-(k+1)}\right)\right) & =\sum_{k=k_{M}}^{\infty} 2^{p k} F_{n}\left(2^{-k}\right)-\sum_{k=1+k_{M}}^{\infty} 2^{p(k-1)} F_{n}\left(2^{-k}\right) \\
& \geq \frac{1}{2} \sum_{k=1+k_{M}}^{\infty} 2^{p k} F_{n}\left(2^{-k}\right),
\end{aligned}
$$

and, since $F_{n}$ is a non-decreasing function,

$$
\begin{aligned}
\int_{0}^{2^{-\left(1+k_{M}\right)}} F_{n}(t) t^{-(p+1)} d t & =\sum_{k=1+k_{M}}^{\infty} \int_{2^{-(k+1)}}^{2^{-k}} F_{n}(t) t^{-(p+1)} d t \\
& \leq \sum_{k=1+k_{M}}^{\infty} F_{n}\left(2^{-k}\right) \int_{2^{-(k+1)}}^{2^{-k}} t^{-(p+1)} d t \\
& \leq \frac{2^{p}}{p} \sum_{k=1+k_{M}}^{\infty} 2^{p k} F_{n}\left(2^{-k}\right) .
\end{aligned}
$$


Thus it follows from 2.21 that

$$
\limsup _{n \rightarrow \infty} 2^{-(p+1)} p \int_{0}^{2^{-\left(1+k_{M}\right)}} F_{n}(t) t^{-(p+1)} d t \leq 2^{-(p+2)} .
$$

This implies

$$
\limsup _{n \rightarrow \infty} \int_{0}^{2^{-\left(1+k_{M}\right)}} F_{n}(t) t^{-(p+1)} d t \leq \frac{1}{2} .
$$

However, by (ii) and (iii), one gets

$$
\lim _{n \rightarrow \infty} \int_{0}^{2^{-\left(1+k_{M}\right)}} F_{n}(t) t^{-(p+1)} d t=1 .
$$

This contradicts 2.22), and proves (2.10). Thus by Proposition 3 it follows that $g \in$ $W^{1, p}\left(\mathbb{R}^{N}\right)$.

In order to prove (1.7), we consider the sequence of functions $G_{n}$ defined by

$$
G_{n}(t)= \begin{cases}F_{n}(t) & \text { if } 0 \leq t \leq 1 \\ F_{n}(1) & \text { otherwise }\end{cases}
$$

This sequence satisfies hypotheses (i)-(iii) of Theorem 1 and (1.8). By assertion (c) of Theorem 1, (1.7) follows.

\subsection{Proof of Theorem 2}

Applying the same method as in Section 2.3, one can prove that

$$
\liminf _{k \rightarrow \infty} \int_{\substack{\mathbb{R}^{N} \\|g(x)-g(y)|>2^{-k}}} \frac{2^{-k}}{|x-y|^{N+1}} d x d y<+\infty
$$

Thus by Proposition 4 one has $g \in B V\left(\mathbb{R}^{N}\right)$ and

$$
\liminf _{k \rightarrow \infty} \int_{\substack{\mathbb{R}^{N} \\|g(x)-g(y)|>2^{-k}}} \frac{2^{-k}}{|x-y|^{N+1}} d x d y \geq c \int_{\mathbb{R}^{N}}|\nabla g| d x .
$$

Hereafter in this proof $c$ denotes a constant depending only on $N$. Thus there exists a constant $k_{0} \in \mathbb{N}$ such that for all $k \geq k_{0}$,

$$
\int_{\mathbb{R}^{N}} \int_{\mathbb{R}^{N}} \frac{2^{-k}}{|x-y|^{N+1}} d x d y \geq c \int_{\mathbb{R}^{N}}|\nabla g| d x .
$$


Applying the method of Section 2.3, one gets

$$
\begin{aligned}
& \int_{\mathbb{R}^{N}} \int_{\mathbb{R}^{N}} \frac{F_{n}(|g(x)-g(y)|)}{|x-y|^{N+1} d x d y \geq c} \int_{0}^{2^{-\left(1+k_{0}\right)}} F_{n}(t) t^{-2} d t \int_{\mathbb{R}^{N}}|\nabla g| d x \\
&+F_{n}(1 / 4) \int_{|g(x)-g(y)|>1 / 2} \int_{\mathbb{R}^{N}} \frac{1}{|x-y|^{N+1}} d x d y .
\end{aligned}
$$

On the other hand, since $F_{n}$ converges uniformly to 0 on every compact subset of $(0,+\infty)$ and

$$
\int_{0}^{1} F_{n}(t) t^{-2} d t=1
$$

it follows that

$$
\lim _{n \rightarrow \infty} \int_{0}^{2^{-\left(1+k_{0}\right)}} F_{n}(t) t^{-2} d t=1
$$

Therefore

$$
\liminf _{n \rightarrow \infty} \int_{\mathbb{R}^{N}} \int_{\mathbb{R}^{N}} \frac{F_{n}(|g(x)-g(y)|)}{|x-y|^{N+1}} d x d y \geq c \int_{\mathbb{R}^{N}}|\nabla g| d x .
$$

Theorems 1 and 2 have the following interesting consequence. It is motivated by the work of J. Bourgain, H. Brezis and P. Mironescu in [1] and [5].

Corollary 1. Let $p \geq 1$ and $\left(F_{n}\right)_{n \in \mathbb{N}}$ be a sequence of non-decreasing functions from $[0,+\infty)$ into $[0,+\infty)$ such that $F_{n}(1)$ is bounded and

$$
\limsup _{n \rightarrow \infty} \int_{0}^{1} F_{n}(t) t^{-(p+1)} d t=+\infty .
$$

Assume that $g \in L^{p}\left(\mathbb{R}^{N}\right)$ and $g$ satisfies 1.6 . Then $g$ is a constant function.

Proof. Without loss of generality, one may assume that

$$
\lim _{n \rightarrow \infty} \int_{0}^{1} F_{n}(t) t^{-(p+1)} d t=+\infty
$$

For each $n \in \mathbb{N}$, set

$$
G_{n}(t)= \begin{cases}\frac{F_{n}(t)}{\int_{0}^{1} F_{n}(t) t^{-(p+1)} d t} & \text { if } 0 \leq t \leq 1, \\ \frac{F_{n}(1)}{\int_{0}^{1} F_{n}(t) t^{-(p+1)} d t} & \text { otherwise. }\end{cases}
$$

Then $\left(G_{n}\right)_{n \in \mathbb{N}}$ satisfies hypotheses (i)-(iii). 
By Theorems 1 and 2 , there exists a constant $c_{N, p}>0$ such that

$$
c_{N, p} \int_{\mathbb{R}^{N}}|\nabla g|^{p} d x \leq \liminf _{n \rightarrow \infty} \int_{\mathbb{R}^{N}} \int_{\mathbb{R}^{N}} \frac{G_{n}(|g(x)-g(y)|)}{|x-y|^{N+p}} d x d y .
$$

However,

$$
\begin{aligned}
\liminf _{n \rightarrow \infty} \int_{\mathbb{R}^{N}} & \int_{\mathbb{R}^{N}} \frac{G_{n}(|g(x)-g(y)|)}{|x-y|^{N+p}} d x d y \\
& \leq \liminf _{n \rightarrow \infty} \frac{1}{\int_{0}^{1} F_{n}(t) t^{-(p+1)} d t} \int_{\mathbb{R}^{N}} \int_{\mathbb{R}^{N}} \frac{F_{n}(|g(x)-g(y)|)}{|x-y|^{N+p}} d x d y=0 .
\end{aligned}
$$

Thus it follows that $\int_{\mathbb{R}^{N}}|\nabla g|^{p} d x=0$. Therefore $g$ is a constant function.

Remark 6. The conditions of Corollary 1 are satisfied by $F_{n}(t)=t^{p}$ for all $n \in \mathbb{N}$ with $p \geq 1$. Hence any function $g \in L^{p}\left(\mathbb{R}^{N}\right)$ satisfying

$$
\iint_{\mathbb{R}^{N} \times \mathbb{R}^{N}} \frac{|g(x)-g(y)|^{p}}{|x-y|^{N+p}} d x d y<+\infty
$$

must be a constant. This was already observed in [5].

\section{Another proof of assertion (b) in Theorem 1}

First in Section 3.1 we present a fundamental lemma. Then in Section 3.2 we discuss a new proof of assertion (b) in Theorem 1

\subsection{A fundamental lemma}

The following lemma will play an important role in this section.

Lemma 2 (Fundamental lemma). Let $g \in L^{p}\left(\mathbb{R}^{N}\right), 1<p<+\infty$. Assume that

$$
\iint_{\substack{K \times K \\|g(x)-g(y)|>\varepsilon}} \frac{1}{|x-y|^{N+1}} d x d y<+\infty, \quad \forall K \subset \subset \mathbb{R}^{N}, \forall \varepsilon>0
$$

and

$$
\liminf _{\varepsilon \rightarrow 0_{+}} \int_{\substack{\mathbb{R}^{N} \times \mathbb{R}^{N} \\ \varepsilon<|g(x)-g(y)|<10 \varepsilon}} \frac{\varepsilon^{p}}{|x-y|^{N+p}} d x d y<+\infty .
$$

Then $g \in W^{1, p}\left(\mathbb{R}^{N}\right)$. 
Remark 7. Condition (3.2) alone is not sufficient to show that $g \in W^{1, p}\left(\mathbb{R}^{N}\right.$ ) (in contrast with condition (1.2)). For example

$$
g(x)= \begin{cases}1 & \text { if }|x|<1, \\ 0 & \text { otherwise }\end{cases}
$$

Surprisingly, the mild additional assumption (3.1) together with (3.2) implies that $g \in W^{1, p}\left(\mathbb{R}^{N}\right)$.

In order to prove Lemma 2, we need some useful lemmas. The first lemma, which was used in [2], is a direct consequence of a result due to J. Bourgain, H. Brezis, and P. Mironescu (see [1]).

Lemma 3. Let $g$ be a measurable function on the interval $[a, b](-\infty<a<b<+\infty)$, $y \in \mathbb{R}$, and $\delta>0$. Set

$$
B=\{x \in[a, b] ; g(x)<y\} .
$$

Assume that

$$
0<\frac{|[a, b] \cap B|}{b-a}<1
$$

and

$$
\int_{|g(x)-g(y)|>\delta}^{b} \int_{a}^{b} \frac{1}{|x-y|^{2}} d x d y<+\infty
$$

Then

$$
\left|[a, b] \cap A_{\tau}\right|>0, \quad \forall \tau>\delta,
$$

where $A_{\tau}:=\{x \in[a, b] ; y \leq g(x)<y+\tau\}$.

Hereafter $|A|$ denotes the Lebesgue measure of $A$ for any measurable set $A \subset \mathbb{R}$.

Proof. We prove Lemma 3 by contradiction. Suppose that $\left|[a, b] \cap A_{\tau}\right|=0$ for some $\tau>\delta$. Then from (3.4),

$$
\int_{B} \int_{[a, b] \backslash B} \frac{1}{|x-y|^{2}} d x d y<+\infty .
$$

This implies (see [1])

$$
|B|=0 \quad \text { or } \quad|[a, b] \backslash B|=0,
$$

which contradicts 3.3 .

The following lemma will be useful to prove Lemma 5 Estimate (3.6) was mentioned and used in [2]. Estimate 3.7] was also hidden there. It will play a role in the proof of Lemma5 For the convenience of the reader, we will reproduce the proof. 
Lemma 4. Let $g$ be a measurable function on the interval $[a, b](-\infty<a<b<+\infty)$, $y \in \mathbb{R}, r>0, s>0$, and $\tau>\delta>0$. Set

$$
B=\{x \in \mathbb{R} ; g(x)<y\}, \quad A=\{x \in \mathbb{R} ; y \leq g(x)<y+\tau\} .
$$

Assume that

$$
\frac{|[a, b] \cap B|}{b-a}=r, \quad \frac{|[a, b] \cap A|}{b-a} \leq s, \quad r+s<1
$$

and

$$
\int_{|g(x)-g(y)|>\delta}^{b} \int_{a}^{b} \frac{1}{|x-y|^{2}} d x d y<+\infty .
$$

Then there exists a subinterval $[c, d] \subset[a, b](a \leq c<d \leq b)$ such that

$$
\frac{|[c, d] \cap B|}{d-c}=r, \quad s / 4 \leq \frac{|[c, d] \cap A|}{d-c} \leq s,
$$

and

$$
\frac{d-c}{b-a} \leq 4 \frac{|[a, b] \cap A|}{s(b-a)} .
$$

Proof. Set $\left[a_{1}, b_{1}\right]=[a, b]$. Suppose that there exists $\left[a_{k}, b_{k}\right] \subset[a, b], k \geq 1$, such that

$$
\frac{\left|\left[a_{k}, b_{k}\right] \cap B\right|}{b_{k}-a_{k}}=r \quad \text { and } \quad \frac{\left|\left[a_{k}, b_{k}\right] \cap A\right|}{b_{k}-a_{k}} \leq s .
$$

If

$$
\frac{\left|\left[a_{k}, b_{k}\right] \cap A\right|}{b_{k}-a_{k}} \geq s / 4
$$

then take $[c, d]=\left[a_{k}, b_{k}\right]$. Otherwise, by Lemma 3 , one has

$$
0<\frac{\left|\left[a_{k}, b_{k}\right] \cap A\right|}{b_{k}-a_{k}}<s / 4 .
$$

Take $s_{k}>0$ such that $s / s_{k} \in \mathbb{Z}_{+}$and

$$
s_{k} / 2<\frac{\left|\left[a_{k}, b_{k}\right] \cap A\right|}{b_{k}-a_{k}} \leq s_{k} .
$$

Then

$$
s_{k}<s / 2
$$

Set

$$
\lambda_{k}=\frac{\left(b_{k}-a_{k}\right) s_{k}}{2 s}
$$

Consider the function $\psi_{k}(t)$ defined as follows:

$$
\psi_{k}(t)=\left|\left[t-\lambda_{k}, t+\lambda_{k}\right] \cap B\right|, \quad \forall t \in\left[a_{k}+\lambda_{k}, b_{k}-\lambda_{k}\right] .
$$

We claim that there exists $t_{k} \in\left[a_{k}+\lambda_{k}, b_{k}-\lambda_{k}\right]$ such that $\psi_{k}\left(t_{k}\right) /\left(2 \lambda_{k}\right)=r$. 
To see this, we argue by contradiction. Suppose that $\psi_{k}(t) /\left(2 \lambda_{k}\right) \neq r$ for all $t \in$ $\left[a_{k}+\lambda_{k}, b_{k}-\lambda_{k}\right]$. Since $\psi_{k}$ is a continuous function on $\left[a_{k}+\lambda_{k}, b_{k}-\lambda_{k}\right]$, we assume as well that $\psi_{k}(t) /\left(2 \lambda_{k}\right)<r$ for all $t \in\left[a_{k}+\lambda_{k}, b_{k}-\lambda_{k}\right]$. Since $\left(b_{k}-a_{k}\right) /\left(2 \lambda_{k}\right)=s / s_{k}$ $\in \mathbb{Z}_{+}$, it follows that

$$
\left|\left[a_{k}, b_{k}\right] \cap B\right|<2 r \lambda_{k} \frac{b_{k}-a_{k}}{2 \lambda_{k}}=r\left(b_{k}-a_{k}\right) .
$$

This contradicts the fact that $\left|\left[a_{k}, b_{k}\right] \cap B\right| /\left(b_{k}-a_{k}\right)=r$.

Set $\left[a_{k+1}, b_{k+1}\right]=\left[t_{k}-\lambda_{k}, t_{k}+\lambda_{k}\right] \subset\left[a_{k}, b_{k}\right]$. Then

$$
\frac{\left|\left[a_{k+1}, b_{k+1}\right] \cap B\right|}{b_{k+1}-a_{k+1}}=r \quad \text { and } \quad \frac{\left|\left[a_{k+1}, b_{k+1}\right] \cap A\right|}{b_{k+1}-a_{k+1}} \leq \frac{\left|\left[a_{k}, b_{k}\right] \cap A\right|}{2 \lambda_{k}} .
$$

Thus it follows from (3.8) and 3.10) that

$$
\frac{\left|\left[a_{k+1}, b_{k+1}\right] \cap A\right|}{b_{k+1}-a_{k+1}} \leq \frac{\left|\left[a_{k}, b_{k}\right] \cap A\right|}{2 \lambda_{k}}=\frac{\left|\left[a_{k}, b_{k}\right] \cap A\right|}{b_{k}-a_{k}} \frac{b_{k}-a_{k}}{2 \lambda_{k}} \leq s_{k} \frac{b_{k}-a_{k}}{2 \lambda_{k}}=s .
$$

Moreover,

Thus from 3.9, this implies

$$
\frac{b_{k+1}-a_{k+1}}{b_{k}-a_{k}}=\frac{2 \lambda_{k}}{b_{k}-a_{k}}=\frac{s_{k}}{s} .
$$

$$
\frac{b_{k+1}-a_{k+1}}{b_{k}-a_{k}} \leq \frac{1}{2}
$$

On the other hand,

$$
\int_{a_{k}}^{b_{k}} \int_{\substack{a_{k} \\|g(x)-g(y)|>\delta}}^{b_{k}} \frac{1}{|x-y|^{2}} d x d y \geq \int_{\substack{x \in\left[a_{k}, b_{k}\right] \cap B \\ y \in\left[a_{k}, b_{k}\right] \backslash(A \cup B)}} \frac{1}{|x-y|^{2}} d x d y,
$$

which shows

$$
\int_{a_{k}}^{b_{k}} \int_{a_{k}}^{b_{k}} \frac{1}{|x-y|^{2}} d x d y \geq r(1-r-s) .
$$

Combining 3.5, 3.11), and 3.12 shows that the above process will stop at some $k \in \mathbb{Z}_{+}$. Then $[c, d] \subset[a, b]\left([c, d]=\left[a_{k}, b_{k}\right]\right)$, and

$$
\frac{|[c, d] \cap B|}{d-c}=r, \quad s / 4 \leq \frac{|[c, d] \cap A|}{d-c} \leq s .
$$

If $k \geq 2$, then it follows from 3.8 and (3.10) that

$$
\frac{d-c}{b-a} \leq \frac{b_{2}-a_{2}}{b_{1}-a_{1}}=\frac{2 \lambda_{1}}{b_{1}-a_{1}}=\frac{s_{1}}{s} \leq 2 \frac{|[a, b] \cap A|}{s(b-a)} .
$$

Otherwise $(k=1)$, the estimate 3.7) holds clearly.

The proof is complete.

The following lemma plays an important role in the proof of Lemma 6 . 
Lemma 5. Let $g$ be a measurable function on the interval $[a, b](-\infty<a<b<+\infty)$, $y \in \mathbb{R}$, and $\tau>\delta>0$. Set

$$
\left\{\begin{array}{l}
B_{j}=\{x \in \mathbb{R} ; g(x)<y+j \tau\}, \\
A_{j}=\{x \in \mathbb{R} ; y+j \tau \leq g(x)<y+(j+1) \tau\},
\end{array} \quad \forall j \in \mathbb{Z} .\right.
$$

Assume that

$$
\frac{\left|[a, b] \cap B_{0}\right|}{b-a}=\frac{1}{2}, \quad \frac{\left|[a, b] \cap A_{0}\right|}{b-a} \leq \frac{1}{8},
$$

and

$$
\int_{|g(x)-g(y)|>\delta}^{b} \int_{a}^{b} \frac{1}{|x-y|^{2}} d x d y<+\infty
$$

Then for each $r>8$, there exist $m \in \mathbb{Z}_{+}, l_{m} \in \mathbb{Z}$, and $[c, d] \subset[a, b](c<d)$ such that

$$
\left\{\begin{array}{l}
\left|l_{m}\right| \leq 2 m, \\
\frac{\left|[c, d] \cap A_{l_{m}}\right|}{d-c} \frac{\left|[c, d] \cap A_{l_{m}+2}\right|}{d-c} \geq \frac{1}{4 \cdot 8^{m+1} r^{m+1}}, \\
d-c \leq 4^{m}(8 / r)^{m(m-1) / 2}(b-a) .
\end{array}\right.
$$

Proof. Set $j_{1}=0$. By Lemma 4, there exists $\left[a_{1}, b_{1}\right] \subset[a, b]$ such that

$$
\frac{\left|\left[a_{1}, b_{1}\right] \cap B_{j_{1}}\right|}{b_{1}-a_{1}}=\frac{1}{2} \text { and } \frac{1}{4 \cdot 8} \leq \frac{\left|\left[a_{1}, b_{1}\right] \cap A_{j_{1}}\right|}{b_{1}-a_{1}} \leq \frac{1}{8} .
$$

Suppose that there exist $\left[a_{k}, b_{k}\right]$ and $j_{k}(k \geq 1)$ such that

$$
\left\{\begin{array}{l}
\left|j_{k}\right| \leq 2(k-1), \\
\frac{1}{2}-2 \sum_{i=1}^{k-1} \frac{1}{8^{i}} \leq \frac{\left|\left[a_{k}, b_{k}\right] \cap B_{j_{k}}\right|}{b_{k}-a_{k}} \leq \frac{1}{2}+2 \sum_{i=1}^{k-1} \frac{1}{8^{i}}, \\
\frac{1}{4 \cdot 8^{k}} \leq \frac{\left|\left[a_{k}, b_{k}\right] \cap A_{j_{k}}\right|}{b_{k}-a_{k}} \leq \frac{1}{8^{k}}
\end{array}\right.
$$

Then we have the following cases:

Case 1:

$$
\frac{\left|\left[a_{k}, b_{k}\right] \cap A_{j_{k}+1}\right|}{b_{k}-a_{k}} \geq \frac{1}{8^{k+1}} \quad \text { and } \quad \frac{\left|\left[a_{k}, b_{k}\right] \cap A_{j_{k}-1}\right|}{b_{k}-a_{k}} \geq \frac{1}{8^{k+1}}
$$

Set

$$
m=k, \quad l_{m}=j_{k}-1, \quad[c, d]=\left[a_{k}, b_{k}\right] .
$$

Then

$$
\frac{\left|[c, d] \cap A_{l_{m}}\right|}{d-c} \frac{\left|[c, d] \cap A_{l_{m}+2}\right|}{d-c} \geq \frac{1}{8^{m+1} 8^{m+1}} \geq \frac{1}{4 \cdot 8^{m+1} r^{m+1}} .
$$

Case 2:

$$
\frac{\left|\left[a_{k}, b_{k}\right] \cap A_{j_{k}+1}\right|}{b_{k}-a_{k}}<\frac{1}{8^{k+1}} \quad \text { or } \quad \frac{\left|\left[a_{k}, b_{k}\right] \cap A_{j_{k}-1}\right|}{b_{k}-a_{k}}<\frac{1}{8^{k+1}}
$$


Case 2.1:

$$
\frac{\left|\left[a_{k}, b_{k}\right] \cap A_{j_{k}+1}\right|}{b_{k}-a_{k}}<\frac{1}{8^{k+1}} .
$$

Case 2.1.1:

$$
\frac{\left|\left[a_{k}, b_{k}\right] \cap A_{j_{k}+2}\right|}{b_{k}-a_{k}} \geq \frac{1}{r^{k+1}} .
$$

Set

$$
m=k, \quad l_{m}=j_{k}, \quad[c, d]=\left[a_{k}, b_{k}\right] .
$$

Then

$$
\frac{\left|[c, d] \cap A_{l_{m}}\right|}{d-c} \frac{\left|[c, d] \cap A_{l_{m}+2}\right|}{d-c} \geq \frac{1}{4 \cdot 8^{m+1} r^{m+1}} .
$$

Case 2.1.2:

$$
\frac{\left|\left[a_{k}, b_{k}\right] \cap A_{j_{k}+2}\right|}{b_{k}-a_{k}}<\frac{1}{r^{k+1}} .
$$

Set

$$
j_{k+1}=j_{k}+2 .
$$

Then, from the first inequality of 3.14,

$$
\left|j_{k+1}\right| \leq 2 k \text {. }
$$

Applying Lemma 4 with $s=1 / 8^{k+1}$ and $B=B_{j_{k+1}}$, one gets $\left[a_{k+1}, b_{k+1}\right] \subset\left[a_{k}, b_{k}\right]$ such that

$$
\left\{\begin{array}{l}
\frac{\left|\left[a_{k+1}, b_{k+1}\right] \cap B_{j_{k+1}}\right|}{b_{k+1}-a_{k+1}}=\frac{\left|\left[a_{k}, b_{k}\right] \cap B_{j_{k+1}}\right|}{b_{k}-a_{k}}, \\
\frac{1}{4 \cdot 8^{k+1}} \leq \frac{\left|\left[a_{k+1}, b_{k+1}\right] \cap A_{j_{k+1}}\right|}{b_{k+1}-a_{k+1}} \leq \frac{1}{8^{k+1}} \\
\frac{b_{k+1}-a_{k+1}}{b_{k}-a_{k}} \leq \frac{4 \cdot 8^{k+1}}{r^{k+1}}
\end{array}\right.
$$

Thus from 3.14 and 3.19,

$$
\frac{\left|\left[a_{k+1}, b_{k+1}\right] \cap B_{j_{k+1}}\right|}{b_{k+1}-a_{k+1}} \geq \frac{\left|\left[a_{k}, b_{k}\right] \cap B_{j_{k}}\right|}{b_{k}-a_{k}} \geq \frac{1}{2}-2 \sum_{i=1}^{k} \frac{1}{8^{i}}
$$

and, since $j_{k+1}=j_{k}+2($ see 3.17$)$,

$$
\begin{aligned}
\frac{\left|\left[a_{k+1}, b_{k+1}\right] \cap B_{j_{k+1}}\right|}{b_{k+1}-a_{k+1}} & =\frac{\left|\left[a_{k}, b_{k}\right] \cap B_{j_{k+1}}\right|}{b_{k}-a_{k}} \\
& =\frac{\left|\left[a_{k}, b_{k}\right] \cap B_{j_{k}}\right|}{b_{k}-a_{k}}+\frac{\left|\left[a_{k}, b_{k}\right] \cap\left(A_{j_{k}} \cup A_{j_{k}+1}\right)\right|}{b_{k}-a_{k}} \\
& \leq \frac{1}{2}+2 \sum_{i=1}^{k-1} \frac{1}{8^{i}}+\frac{1}{8^{k}}+\frac{1}{8^{k+1}} .
\end{aligned}
$$


Hence

$$
\frac{1}{2}-2 \sum_{i=1}^{k} \frac{1}{8^{i}} \leq \frac{\left|\left[a_{k+1}, b_{k+1}\right] \cap B_{j_{k+1}}\right|}{b_{k+1}-a_{k+1}} \leq \frac{1}{2}+2 \sum_{i=1}^{k} \frac{1}{8^{i}} .
$$

Therefore from 3.18, 3.20) and the last two estimates of 3.19), one gets

$$
\left\{\begin{array}{l}
\left|j_{k+1}\right| \leq 2 k \\
\frac{1}{2}-2 \sum_{i=1}^{k} \frac{1}{8^{i}} \leq \frac{\left|\left[a_{k+1}, b_{k+1}\right] \cap B_{j_{k+1}}\right|}{b_{k+1}-a_{k+1}} \leq \frac{1}{2}+2 \sum_{i=1}^{k} \frac{1}{8^{i}} \\
\frac{1}{4 \cdot 8^{k+1}} \leq \frac{\left|\left[a_{k+1}, b_{k+1}\right] \cap A_{j_{k+1}}\right|}{b_{k+1}-a_{k+1}} \leq \frac{1}{8^{k+1}} \\
\frac{b_{k+1}-a_{k+1}}{b_{k}-a_{k}} \leq \frac{4 \cdot 8^{k+1}}{r^{k+1}}
\end{array}\right.
$$

Case 2.2:

$$
\frac{\left|\left[a_{k}, b_{k}\right] \cap A_{j_{k}+1}\right|}{b_{k}-a_{k}} \geq \frac{1}{8^{k+1}} \quad \text { and } \quad \frac{\left|\left[a_{k}, b_{k}\right] \cap A_{j_{k}-1}\right|}{b_{k}-a_{k}}<\frac{1}{8^{k+1}}
$$

Case 2.2.1:

$$
\frac{\left|\left[a_{k}, b_{k}\right] \cap A_{j_{k}-2}\right|}{b_{k}-a_{k}} \geq \frac{1}{r^{k+1}} .
$$

Set

$$
m=k, \quad l_{m}=j_{k}-2, \quad[c, d]=\left[a_{k}, b_{k}\right]
$$

Then

$$
\frac{\left|[c, d] \cap A_{l_{m}}\right|}{d-c} \frac{\left|[c, d] \cap A_{l_{m}+2}\right|}{d-c} \geq \frac{1}{4 \cdot 8^{m+1} r^{m+1}} .
$$

Case 2.2.2:

$$
\frac{\left|\left[a_{k}, b_{k}\right] \cap A_{j_{k}-2}\right|}{b_{k}-a_{k}}<\frac{1}{r^{k+1}}
$$

Set

$$
j_{k+1}=j_{k}-2
$$

Then from the first inequality of 3.14,

$$
\left|j_{k+1}\right| \leq 2 k
$$

Applying Lemma 4 with $s=1 / 8^{k+1}$ and $B=B_{j_{k+1}}$, one gets $\left[a_{k+1}, b_{k+1}\right] \subset\left[a_{k}, b_{k}\right]$ such that

$$
\left\{\begin{array}{l}
\frac{\left|\left[a_{k+1}, b_{k+1}\right] \cap B_{j_{k+1}}\right|}{b_{k+1}-a_{k+1}}=\frac{\left|\left[a_{k}, b_{k}\right] \cap B_{j_{k+1}}\right|}{b_{k}-a_{k}} \\
\frac{1}{4 \cdot 8^{k+1}} \leq \frac{\left|\left[a_{k+1}, b_{k+1}\right] \cap A_{j_{k+1}}\right|}{b_{k+1}-a_{k+1}} \leq \frac{1}{8^{k+1}} \\
\frac{b_{k+1}-a_{k+1}}{b_{k}-a_{k}} \leq \frac{4 \cdot 8^{k+1}}{r^{k+1}}
\end{array}\right.
$$


Thus from the second estimate of 3.14,

$$
\frac{\left|\left[a_{k+1}, b_{k+1}\right] \cap B_{j_{k+1}}\right|}{b_{k+1}-a_{k+1}} \leq \frac{\left|\left[a_{k}, b_{k}\right] \cap B_{j_{k}}\right|}{b_{k}-a_{k}} \leq \frac{1}{2}+2 \sum_{i=1}^{k} \frac{1}{8^{i}} .
$$

and, since $j_{k+1}=j_{k}-2($ see 3.23$)$,

$$
\begin{aligned}
\frac{\left|\left[a_{k+1}, b_{k+1}\right] \cap B_{j_{k+1}}\right|}{b_{k+1}-a_{k+1}} & =\frac{\left|\left[a_{k}, b_{k}\right] \cap B_{j_{k+1}}\right|}{b_{k}-a_{k}} \\
& =\frac{\left|\left[a_{k}, b_{k}\right] \cap B_{j_{k}}\right|}{b_{k}-a_{k}}-\frac{\left|\left[a_{k}, b_{k}\right] \cap\left(A_{j_{k}-1} \cup A_{j_{k}-2}\right)\right|}{b_{k}-a_{k}} \\
& \geq \frac{1}{2}-2 \sum_{i=1}^{k-1} \frac{1}{8^{i}}-\frac{1}{8^{k+1}}-\frac{1}{r^{k+1}} .
\end{aligned}
$$

Hence

$$
\frac{1}{2}-2 \sum_{i=1}^{k} \frac{1}{8^{i}} \leq \frac{\left|\left[a_{k+1}, b_{k+1}\right] \cap B_{j_{k+1}}\right|}{b_{k+1}-a_{k+1}} \leq \frac{1}{2}+2 \sum_{i=1}^{k} \frac{1}{8^{i}} .
$$

Therefore from (3.24), 3.26) and the last two estimates of 3.25, one has

$$
\left\{\begin{array}{l}
\left|j_{k+1}\right| \leq 2 k \\
\frac{1}{2}-2 \sum_{i=1}^{k} \frac{1}{8^{i}} \leq \frac{\left|\left[a_{k+1}, b_{k+1}\right] \cap B_{j_{k+1}}\right|}{b_{k+1}-a_{k+1}} \leq \frac{1}{2}+2 \sum_{i=1}^{k} \frac{1}{8^{i}} \\
\frac{1}{4 \cdot 8^{k+1}} \leq \frac{\left|\left[a_{k+1}, b_{k+1}\right] \cap A_{j_{k+1}}\right|}{b_{k+1}-a_{k+1}} \leq \frac{1}{8^{k+1}} \\
\frac{b_{k+1}-a_{k+1}}{b_{k}-a_{k}} \leq \frac{4 \cdot 8^{k+1}}{r^{k+1}}
\end{array}\right.
$$

On the other hand, from 3.14,

$$
\int_{\substack{a_{k} \\|g(x)-g(y)|>\delta}}^{b_{k}} \int_{\substack{a_{k} \\ b_{k}}} \frac{1}{|x-y|^{2}} d x d y \geq \int_{\substack{x \in\left[a_{k}, b_{k}\right] \cap B_{j_{k}} \\ y \in\left[a_{k}, b_{k}\right] \backslash\left(B_{j_{k}} \cup A_{j_{k}}\right)}} \frac{1}{|x-y|^{2}} d x d y \gtrsim 1 .
$$

Thus it follows from inequality (3.28), the last inequalities of (3.21) and (3.27), and (3.13) that this process will stop at some $k \in \mathbb{Z}_{+}$. Thus from 3.15, 3.16 and 3.22, it suffices to show that $\left([c, d]=\left[a_{m}, b_{m}\right]\right)$

$$
d-c \leq 4^{m}(8 / r)^{m(m-1) / 2}(b-a) .
$$

In fact, from the last inequality of 3.21 and 3.27,

$$
b_{i}-a_{i} \leq \frac{4 \cdot 8^{i}}{r^{i}}\left(b_{i-1}-a_{i-1}\right), \quad \forall 2 \leq i \leq m,
$$


which shows, since $d-c=b_{m}-a_{m}$,

$$
d-c \leq 4^{m}(8 / r)^{m(m+1) / 2-1}(b-a) \leq 4^{m}(8 / r)^{m(m-1) / 2}(b-a)
$$

(the above inequality is evident when $m=1$ ).

The proof is complete.

Lemma 5 has the following consequence which is one of the main ingredients to establish Lemma6,

Corollary 2. Let $1<p<+\infty$. Under the hypotheses of Lemma 5 there exist $m \in \mathbb{Z}_{+}$ and $l_{m} \in \mathbb{Z}$ such that

$$
\left|l_{m}\right| \leq 2 m
$$

and

$$
\iint_{\substack{x \in[a, b] \cap A_{l m} \\ y \in[a, b] \cap A_{l_{m}+2}}} \frac{1}{|x-y|^{p+1}} d x d y \geq c_{p} m(b-a)^{1-p}
$$

for some positive constant $c_{p}$ depending only on $p$.

Proof. Take $r=16$. By Lemma 5 , there exist $m \in \mathbb{Z}_{+}, l_{m} \in \mathbb{Z}$, and $[c, d] \subset[a, b]$ such that

$$
\left\{\begin{array}{l}
\left|l_{m}\right| \leq 2 m, \\
\frac{\left|[c, d] \cap A_{l_{m}}\right|}{d-c} \frac{\left|[c, d] \cap A_{l_{m}+2}\right|}{d-c} \geq \frac{1}{4 \cdot 8^{m+1} r^{m+1}}, \\
d-c \leq 4^{m}(8 / r)^{m(m-1) / 2}(b-a) .
\end{array}\right.
$$

Hence

$$
\begin{aligned}
\frac{\left|[c, d] \cap A_{l_{m}}\right|\left|[c, d] \cap A_{l_{m}+2}\right|}{(d-c)^{p+1}} & \geq \frac{1}{4 \cdot 8^{m+1} r^{m+1}}(d-c)^{1-p} \\
& \geq \frac{1}{4 \cdot 8^{m+1} r^{m+1}} 4^{m(1-p)}(r / 8)^{m(m-1)(p-1) / 2}(b-a)^{1-p},
\end{aligned}
$$

which shows that (since $p>1$ )

$$
\frac{\left|[c, d] \cap A_{l_{m}}\right|\left|[c, d] \cap A_{l_{m}+2}\right|}{(d-c)^{p+1}} \geq c_{p} m(b-a)^{1-p}
$$

for some positive constant $c_{p}$ depending only on $p$.

On the other hand,

$$
\iint_{\substack{x \in[c, d] \cap A_{l_{m}} \\ y \in[c, d] \cap A_{l_{m}+2}}} \frac{1}{|x-y|^{p+1}} d x d y \geq \frac{\left|[c, d] \cap A_{l_{m}}\right|\left|[c, d] \cap A_{l_{m}+2}\right|}{(d-c)^{p+1}} .
$$


Therefore,

$$
\iint_{\substack{x \in[a, b] \cap A_{l_{m}} \\ y \in[a, b] \cap A_{l_{m}+2}}} \frac{1}{|x-y|^{p+1}} d x d y \geq c_{p} m(b-a)^{1-p} .
$$

The following lemma plays a crucial role in this section.

Lemma 6. Let $g$ be a measurable function on a bounded interval $\mathcal{I}$ and $1<p<+\infty$. Assume that

$$
\iint_{\substack{\mathcal{I} \times \mathcal{I} \\(x)-g(y) \mid>\varepsilon}} \frac{1}{|x-y|^{2}} d x d y<+\infty, \quad \forall \varepsilon>0 .
$$

Then

$$
\liminf _{\varepsilon \rightarrow 0_{+}} \int_{\substack{\mathcal{I} \times \mathcal{I} \\ \varepsilon<|g(x)-g(y)|<10 \varepsilon}} \frac{\varepsilon^{p}}{|x-y|^{p+1}} d x d y \geq c_{p} \frac{1}{|\mathcal{I}|^{p-1}}(\underset{\mathcal{I}}{\operatorname{ess} \sup } g-\underset{\mathcal{I}}{\operatorname{essinf}} g)^{p}
$$

for some positive constant $c_{p}$ depending only on $p$.

Proof. Case 1: $f$ is bounded. We first follow the idea and the notations used in the proof of [2, Lemma 1].

By rescaling, we may assume $\mathcal{I}=[0,1]$. Set $s_{+}=\operatorname{ess}_{\sup } g, s_{-}=\operatorname{essinf}_{\mathcal{I}} g$. Rescaling and translating $g$, one may also assume

$$
s_{+}=1 \quad \text { and } \quad s_{-}=0
$$

(unless $g$ is constant on $\mathcal{I}$ in which case there is nothing to prove).

Take $0<\delta \ll 1$ small enough to ensure that there are (density) points $t_{+}, t_{-} \in$ $[40 \delta, 1-40 \delta] \subset[0,1]$ with

$$
\left\{\begin{array}{l}
\left|\left[t_{+}-\tau, t_{+}+\tau\right] \cap\left[g>\frac{3}{4}\right]\right|>\frac{9}{5} \tau, \quad \forall 0<\tau<40 \delta . \\
\left|\left[t_{-}-\tau, t_{-}+\tau\right] \cap\left[g<\frac{1}{4}\right]\right|>\frac{9}{5} \tau,
\end{array}\right.
$$

Take $K \in \mathbb{Z}_{+}$such that

$$
\delta<2^{-K} \leq 2 \delta
$$

and define

$$
J=\left\{j \in \mathbb{Z}_{+} ; 1 / 4<j 2^{-K}<3 / 4\right\} .
$$

Then

$$
|J| \geq 2^{K-1}-2 \approx 1 / \delta
$$


For each $j$, set

$$
A_{j}=\left\{x \in[0,1] ;(j-1) 2^{-K} \leq g(x)<j 2^{-K}\right\} \quad \text { and } \quad B_{j}=\bigcup_{j^{\prime}<j} A_{j^{\prime}} .
$$

Since $A_{j} \cap A_{k}=\emptyset$ for $j \neq k$, it follows from 3.35 that

$$
\operatorname{card}(G) \geq 2^{K-2}-3 \approx 1 / \delta, \quad \text { where } \quad G=\left\{j \in J ;\left|A_{j}\right|<2^{-K+2}\right\} .
$$

For each $j \in G$, set $\lambda_{j}=\left|A_{j}\right|$ and consider the function $\psi_{j}(t)$ defined as follows:

$$
\psi_{j}(t)=\left|\left[t-4 \lambda_{j}, t+4 \lambda_{j}\right] \cap B_{j}\right|, \quad \forall t \in[40 \delta, 1-40 \delta] .
$$

Then, from 3.33,

$$
\psi_{j}\left(t_{+}\right)<4 \lambda_{j} \text { and } \quad \psi_{j}\left(t_{-}\right)>4 \lambda_{j}
$$

Thus, since $\psi_{j}$ is a continuous function on the interval [40, $1-40 \delta$ ] containing two points $t_{+}$and $t_{-}$, there exists $t_{j} \in[40 \delta, 1-40 \delta]$ such that

$$
\psi_{j}\left(t_{j}\right)=4 \lambda_{j}
$$

In the rest of the proof we introduce a new way to estimate the left side of (3.31). Since $\lambda_{j} \lesssim \delta$, it follows from Corollary 2 that there exist $m_{j} \in \mathbb{Z}_{+}$and $l_{j} \in \mathbb{Z}$ such that

$$
\left|l_{j}-j\right| \leq 2 m_{j}
$$

and

$$
\iint_{\substack{x \in \mathcal{I} \cap A_{l_{j}} \\ y \in \mathcal{I} \cap A_{l_{j}+2}}} \frac{1}{|x-y|^{p+1}} d x d y \geq c_{p} m_{j} \delta^{1-p},
$$

for some positive constant $c_{p}$ depending only on $p$.

Set $i_{0}=-1$ and

$$
C_{i}=\left\{j \in G ; l_{j}=i\right\}, \quad \forall i \in \mathbb{Z} .
$$

For each $n \geq 1$, if

$$
\left\{i \in \mathbb{Z} ; i \geq i_{n-1}+1 \text { and } C_{i} \neq \emptyset\right\} \neq \emptyset
$$

then set

$$
\begin{aligned}
& i_{n}=\inf \left\{i \in \mathbb{Z} ; i \geq i_{n-1}+1 \text { and } C_{i} \neq \emptyset\right\}, \\
& k_{n}=\max \left\{m_{j} ; j \in G \text { and } l_{j}=i_{n}\right\} .
\end{aligned}
$$

Then

$$
k_{n} \gtrsim \operatorname{card}\left\{j \in G ; l_{j}=i_{n}\right\} .
$$

Hence it follows from 3.36 that

$$
\sum_{n \geq 1, k_{n} \text { exists }} k_{n} \gtrsim \operatorname{card}(G) \approx \frac{1}{\delta} .
$$


On the other hand, from 3.37,

$$
\begin{aligned}
& \underset{\substack{\mathcal{I} \\
2^{-K} \leq|g(x)-g(y)| \leq 3 \cdot 2^{-K}}}{\int_{\mathcal{I}}} \frac{\delta^{p}}{|x-y|^{p-1}} d x d y \geq \sum_{n \geq 1, k_{n} \text { exists }} \iint_{\substack{x \in \mathcal{I} \cap A_{i_{n}} \\
y \in \mathcal{I} \cap A_{i_{n}+2}}} \frac{\delta^{p}}{|x-y|^{p+1}} d x d y \\
& \geq c_{p} \sum_{n \geq 1, k_{n} \text { exists }} k_{n} \delta .
\end{aligned}
$$

Therefore the conclusion of Lemma 6 follows from 3.38, 3.39, and 3.34).

Case 2: $f$ is unbounded. By the method used in Case 1, one has

$$
\liminf _{\varepsilon \rightarrow 0_{+}} \iint_{\substack{\mathcal{I} \times \mathcal{I} \\ \varepsilon<|g(x)-g(y)|<10 \varepsilon}} \frac{\varepsilon^{p}}{|x-y|^{p+1}} d x d y=+\infty .
$$

Remark 8. It is interesting to compare Lemma 6 with Lemma 2 in [2] which asserts that, for each $p \geq 1$, there exists a positive constant $c_{p}$ depending only on $p$ such that, for any bounded interval $\mathcal{I}$ and for any measurable function $g$ defined on $\mathcal{I}$,



Obviously, Lemma 6 implies this assertion for the case $p>1$.

We are now ready to prove Lemma2

Proof of Lemma 2. Without loss of generality, we assume that

$$
\sup _{n \in \mathbb{N}} \int_{\substack{\mathbb{R}^{N} \times \mathbb{R}^{N} \\ \varepsilon_{n}<|g(x)-g(y)|<10 \varepsilon_{n}}} \frac{\varepsilon_{n}^{p}}{|x-y|^{p+N}} d x d y<+\infty
$$

for some sequence of positive numbers $\varepsilon_{n}$ such that $\lim _{n \rightarrow \infty} \varepsilon_{n}=0$.

Step 1: Proof of Lemma 2 when $N=1$. This proof is similar to the one of [2, Theorem 1] for the case $N=1$. We reproduce it here for the convenience of the reader.

Set

$$
\tau_{h}(g)(x)=\frac{g(x+h)-g(x)}{h}, \quad \forall x \in \mathbb{R}, \forall 0<h<1 .
$$

For each $m \geq 2$, take $K \in \mathbb{R}_{+}$such that $K h>m$; then

$$
\int_{-m}^{m}\left|\tau_{h}(g)(x)\right|^{p} d x \leq \sum_{k=-K}^{K} \int_{k h}^{(k+1) h}\left|\tau_{h}(g)(x)\right|^{p} d x .
$$


Thus, since

$$
\int_{a}^{a+h}\left|\tau_{h}(g)(x)\right|^{p} d x \leq \int_{a}^{a+h} \frac{1}{h^{p}}|\underset{x \in(a, a+2 h)}{\operatorname{ess} \sup } g-\underset{x \in(a, a+2 h)}{\operatorname{ess} \inf } g|^{p} d x,
$$

it follows from Lemma 6 that, for some constant $c_{p}>0$,

$$
\int_{-m}^{m}\left|\tau_{h}(g)(x)\right|^{p} d x \leq c_{p} \sup _{n \in \mathbb{N}} \int_{\substack{\mathbb{R} \times \mathbb{R} \\ \varepsilon_{n}<|g(x)-g(y)|<10 \varepsilon_{n}}} \frac{\varepsilon_{n}^{p}}{|x-y|^{p+1}} d x d y .
$$

Since $m \geq 2$ is arbitrary, we deduce from 3.41, that

$$
\int_{\mathbb{R}}\left|\tau_{h}(g)(x)\right|^{p} d x \leq c_{p} \sup _{n \in \mathbb{N}} \iint_{\substack{\mathbb{R} \times \mathbb{R} \\ \varepsilon_{n}<|g(x)-g(y)|<10 \varepsilon_{n}}} \frac{\varepsilon_{n}^{p}}{|x-y|^{p+1}} d x d y .
$$

Therefore since (3.42) holds for all $0<h<1$, it follows that $g \in W^{1, p}(\mathbb{R})$ (see [4] Chapter 8]).

Step 2: Proof of Theorem 1 for $N \geq 2$. Using the change of variables formula and Fubini's theorem, one gets

$$
\int_{\mathbb{R}^{N}} \int_{\mathbb{R}^{N}} \frac{1}{|x-y|^{p+N}} d x d y=\int_{\substack{\mathbb{S}^{N-1} \\ \varepsilon<|g(x+\sigma h)-g(x)|<10 \varepsilon}} \int_{\mathbb{R}^{N}} \int_{0}^{\infty} \frac{1}{h^{p+1}} d h d x d \sigma .
$$

Hence, it follows from 3.2 that

$$
\liminf _{\varepsilon \rightarrow 0_{+}} \int_{\varepsilon<|g(x+\sigma h)-g(x)|<10 \varepsilon} \int_{\mathbb{S}^{N-1}} \int_{\mathbb{R}^{N}}^{\infty} \frac{\varepsilon^{p}}{h^{p+1}} d h d x d \sigma<+\infty .
$$

Applying Fatou's lemma, one has

$$
\int_{\mathbb{S}^{N-1}} \liminf _{\varepsilon \rightarrow 0_{+}} \int_{\varepsilon<|g(x+\sigma h)-g(x)|<10 \varepsilon} \int_{\mathbb{R}^{N}}^{\infty} \frac{\varepsilon^{p}}{h^{p+1}} d h d x d \sigma<+\infty
$$

Thus for almost every $\sigma \in \mathbb{S}^{N-1}$,

$$
\liminf _{\varepsilon \rightarrow 0_{+}} \int_{\varepsilon<|g(x+\sigma h)-g(x)|<10 \varepsilon} \int_{\mathbb{R}^{N}}^{\infty} \frac{\varepsilon^{p}}{h^{p+1}} d h d x<+\infty .
$$

On the other hand, from 3.1,

$$
\int_{\substack{\mathbb{S}^{N-1} \\|g(x+h \sigma)-g(x)|>\varepsilon}} \int_{\substack{|x|<r \\ \mid}}^{r} \frac{1}{h^{p+1}} d h d x d \sigma<+\infty, \quad \forall r>0, \forall \varepsilon>0 .
$$


Hence

$$
\int_{\substack{|x|<r \\ g(x+h \sigma)-g(x) \mid>\varepsilon}} \int_{0}^{r} \frac{1}{h^{p+1}} d h d x<+\infty, \quad \forall r>0, \forall \varepsilon>0,
$$

for almost every $\sigma \in \mathbb{S}^{N-1}$.

Fix $\sigma \in \mathbb{S}^{N-1}$ such that conditions 3.43 and 3.44 are satisfied. We claim that

$$
\frac{\partial g}{\partial \sigma} \in L^{p}\left(\mathbb{R}^{N}\right)
$$

In fact, without loss of generality, suppose that $\sigma=e_{N}:=(0, \ldots, 0,1)$. Then from (3.43, we have

$$
\liminf _{\varepsilon \rightarrow 0} \int_{\varepsilon<\left|g\left(x^{\prime}, x_{N}+h\right)-g\left(x^{\prime}, x_{N}\right)\right|<10 \varepsilon} \int_{\mathbb{R}^{N-1}}^{\infty} \frac{\varepsilon^{p}}{h^{p+1}} d h d x_{N} d x^{\prime}<+\infty .
$$

Hence applying Fatou's lemma, one gets

$$
\int_{\mathbb{R}^{N-1}} \liminf _{\varepsilon \rightarrow 0} \int_{\mathbb{R}} \int_{\mathbb{R}^{\prime}\left|g\left(x^{\prime}, x_{N}\right)-g\left(x^{\prime}, y_{N}\right)\right|<10 \varepsilon} \frac{\varepsilon^{p}}{\left|x_{N}-y_{N}\right|^{p+1}} d x_{N} d y_{N} d x^{\prime}<+\infty .
$$

On the other hand, 3.44 gives

$$
\iint_{\substack{K \times K \\\left|g\left(x^{\prime}, x_{N}\right)-g\left(x^{\prime}, y_{N}\right)\right|>\varepsilon}} \frac{\varepsilon^{p}}{\left|x_{N}-y_{N}\right|^{p+1}} d x_{N} d y_{N}<+\infty, \quad \forall K \subset \subset \mathbb{R}, \forall \varepsilon>0,
$$

for almost every $x^{\prime} \in \mathbb{R}^{N-1}$. Therefore applying Lemma 2 for the case $N=1$, one has $g\left(x^{\prime}, \cdot\right) \in W^{1, p}(\mathbb{R})$ for almost every $x^{\prime} \in \mathbb{R}^{N-1}$ and moreover (see [7]),

$$
\int_{\mathbb{R}^{N}}\left|\frac{\partial g}{\partial x_{N}}(x)\right|^{p} d x=\int_{\mathbb{R}^{N-1}} \int_{\mathbb{R}}\left|\frac{\partial g}{\partial x_{N}}\left(x^{\prime}, x_{N}\right)\right|^{p} d x_{N} d x^{\prime}<+\infty .
$$

Since $\partial g / \partial \sigma \in L^{p}\left(\mathbb{R}^{N}\right)$ for almost every $\sigma \in \mathbb{S}^{N-1}$, we conclude that $g \in W^{1, p}\left(\mathbb{R}^{N}\right)$.

This completes the proof of the fundamental lemma 2

Remark 9. The constant 10 which appears in the condition " $\varepsilon<|g(x)-g(y)|<$ $10 \varepsilon$ " is a technical constant. We believe that 10 can be replaced by any positive constant strictly greater than 1 , but we have not been able to prove this.

Remark 10. Lemma 2 is only proved in the case $1<p<+\infty$. Lemma 2 clearly implies Proposition 3 .

When $p=1$ we have the following 
Question 2. Assume that $g \in L^{1}\left(\mathbb{R}^{N}\right)$ satisfies

$$
\iint_{\substack{K \times K \\(x)-g(y) \mid>\varepsilon}} \frac{1}{|x-y|^{N+1}} d x d y<+\infty, \quad \forall K \subset \subset \mathbb{R}^{N}, \forall \varepsilon>0
$$

and

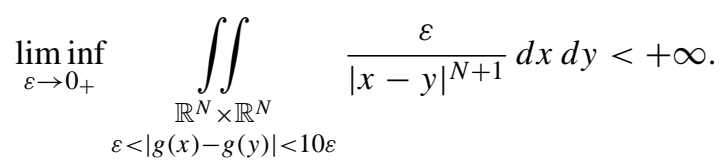

Does g belong to $B V\left(\mathbb{R}^{N}\right)$ ?

\subsection{Proof of assertion (b) in Theorem 1}

Without loss of generality, we may assume that

$$
M:=1+\sup _{n \in \mathbb{N}} \int_{\mathbb{R}^{N}} \int_{\mathbb{R}^{N}} \frac{F_{n}(|g(x)-g(y)|)}{|x-y|^{p+N}} d x d y<+\infty .
$$

Thus since $F_{n}(t)$ is a non-decreasing function with respect to $t$,

$$
\sup _{n \in \mathbb{N}} \sum_{k=1}^{\infty} 10^{p(k+1)} F_{n}\left(10^{-(k+1)}\right) \underset{\substack{0^{-(k+1)}<|g(x)-g(y)|<10^{-k} \\ \int_{\mathbb{R}^{N}}}}{\int_{\mathbb{R}^{N}}} \frac{10^{-p(k+1)}}{|x-y|^{p+N}} d x d y \leq M .
$$

We claim that

$$
\liminf _{k \rightarrow \infty} \int_{\substack{\mathbb{R}^{N} \\ 10^{-(k+1)}<|g(x)-g(y)|<10^{-k}}} \int_{\mathbb{R}^{N}} \frac{10^{-p(k+1)}}{|x-y|^{p+N}} d x d y<+\infty .
$$

We prove (3.46) by contradiction. Suppose it does not hold. Then there exists $k_{M} \in \mathbb{N}$ such that

$$
\int_{\mathbb{R}^{N}} \int_{\mathbb{R}^{N}} \frac{10^{-p(k+1)}}{10^{-(k+1)}<|g(x)-g(y)|<10^{-k}} d x d y>2 \cdot 10^{p+N} d x, \quad \forall k \geq k_{M} .
$$

Hence (3.45) implies

$$
\sup _{n \in \mathbb{N}} 2 \cdot 10^{p+1} M \sum_{k=k_{M}}^{\infty} 10^{p(k+1)} F_{n}\left(10^{-(k+1)}\right) \leq M,
$$

which shows that

$$
\sup _{n \in \mathbb{N}} \sum_{k=k_{M}}^{\infty} 10^{-(k+1)} 10^{(k+2)(p+1)} F_{n}\left(10^{-(k+1)}\right) \leq \frac{1}{2} .
$$


On the other hand, since $F_{n}$ converges uniformly to 0 on any compact subset of $(0,+\infty)$ and

$$
\int_{0}^{1} F_{n}(t) t^{-(p+1)} d t=1
$$

(see hypotheses (ii) and (iii) on $F_{n}$ ), one gets

$$
\begin{aligned}
1=\lim _{n \rightarrow \infty} \int_{0}^{10^{-\left(k_{M}+1\right)}} F_{n}(t) t^{-(p+1)} d t & =\lim _{n \rightarrow \infty} \sum_{k=k_{M}}^{\infty} \int_{10^{-(k+2)}}^{10^{-(k+1)}} F_{n}(t) t^{-(p+1)} d t \\
& \leq \limsup _{n \rightarrow \infty} \sum_{k=k_{M}}^{\infty} 10^{-(k+1)} 10^{(k+2)(p+1)} F_{n}\left(10^{-(k+1)}\right)
\end{aligned}
$$

(since $F_{n}(t)$ is a non-decreasing function with respect to $t$ ). This contradicts 3.47), and proves (3.46).

By (i)-(iii) it follows that for each $s>0$ there exists $n$ such that $F_{n}(s)>0$. Hence since $F_{n}$ is a non-decreasing function,

$$
\int_{\mathbb{R}^{N}} \int_{\mathbb{R}^{N}} \frac{1}{|x-y|^{p+N}} d x d y<+\infty, \quad \forall s>0 .
$$

Therefore by Lemma 2 , it follows from 3.46 and 3.48 that $g \in W^{1, p}\left(\mathbb{R}^{N}\right)$.

\section{4. $\Gamma$-convergence}

In this section we investigate some questions relating to $\Gamma$-convergence. In [8], A. C. Ponce studied similar questions in the context of [1].

We first recall the concept of $\Gamma$-convergence (see [3, 6]). One says that a sequence of functionals $\left(I_{n}\right)$, with values in $[0,+\infty], \Gamma$-converges to a functional $I$ on $L^{p}\left(\mathbb{R}^{N}\right)$ when the following two conditions are satisfied:

(A) For every $g \in L^{p}\left(\mathbb{R}^{N}\right)$, there exists a sequence $\left(g_{n}\right)_{n \in \mathbb{N}} \subset L^{p}\left(\mathbb{R}^{N}\right)$ converging to $g$ in $L^{p}\left(\mathbb{R}^{N}\right)$ and

$$
\limsup _{n \rightarrow \infty} I_{n}\left(g_{n}\right) \leq I(g) .
$$

(B) For every $g \in L^{p}\left(\mathbb{R}^{N}\right)$ and for every sequence $\left(g_{n}\right)_{n \in \mathbb{N}} \subset L^{p}\left(\mathbb{R}^{N}\right)$ converging to $g$ in $L^{p}\left(\mathbb{R}^{N}\right)$, we have

$$
I(g) \leq \liminf _{n \rightarrow \infty} I_{n}\left(g_{n}\right)
$$

We now take

$$
I_{p, n}(g)=\int_{\substack{\mathbb{R}^{N} \times \mathbb{R}^{N} \\|g(x)-g(y)|>\delta_{n}}} \frac{\delta_{n}^{p}}{|x-y|^{N+p}} d x d y
$$


for some sequence $\left(\delta_{n}\right)$ converging to 0 , and

$$
I_{p}(g)=\left\{\begin{aligned}
\frac{K_{N, p}}{p} \int_{\mathbb{R}^{N}}|\nabla g|^{p} d x & \\
& \text { if } g \in W^{1, p}\left(\mathbb{R}^{N}\right) \text { for } p>1, \text { resp. } g \in B V\left(\mathbb{R}^{N}\right) \text { for } p=1, \\
+\infty \quad & \text { otherwise. }
\end{aligned}\right.
$$

When $1<p<\infty$, property (A) is satisfied with $g_{n}=g$ by Proposition 1. A very interesting open question is

Question 3. When $1<p<\infty$, does $\left(I_{p, n}\right) \Gamma$-converge to $I_{p}$ on $L^{p}\left(\mathbb{R}^{N}\right)$ ?

In order to give a positive answer to Question 3 , it would suffice to prove property (B), i.e., that one can take $c_{N, p}=(1 / p) K_{N, p}$ in 4.3 below. This problem is open even for $N=1$. A partial answer is given in Theorem 3 below.

The same question can be asked for $p=1$ :

Question 4. Does $\left(I_{1, n}\right) \Gamma$-converge to $I_{1}$ on $L^{1}\left(\mathbb{R}^{N}\right)$ ?

Here the situation is more delicate. As pointed out in Remark 5, there exists a function $g \in W^{1,1}(\mathbb{R})$ such that $\lim _{n \rightarrow \infty} I_{1, n}(g)=+\infty$ (while $\left.I_{1}(g)<+\infty\right)$. Hence we cannot argue as above by taking $g_{n}=g$ to prove property (A). However, (A) is still true:

Proposition 5. Given any $g \in B V\left(\mathbb{R}^{N}\right)$ there exists a sequence $g_{n} \in C_{c}^{\infty}\left(\mathbb{R}^{N}\right)$ converging to $g$ in $L^{1}\left(\mathbb{R}^{N}\right)$ and such that

$$
\limsup _{n \rightarrow \infty} I_{1, n}\left(g_{n}\right) \leq K_{N, 1} \int_{\mathbb{R}^{N}}|\nabla g| d x .
$$

Proof. Let $\left(h_{k}\right)_{k \in \mathbb{N}}$ be a sequence in $C_{c}^{\infty}\left(\mathbb{R}^{N}\right)$ converging to $g$ in $L^{1}\left(\mathbb{R}^{N}\right)$ and such that

$$
\lim _{k \rightarrow \infty} \int_{\mathbb{R}^{N}}\left|\nabla h_{k}\right| d x=\int_{\mathbb{R}^{N}}|\nabla g| d x
$$

Using the same method as in the proof of [7, Lemma 3], one gets

$$
\lim _{\delta \rightarrow 0} I_{1}\left(h_{k}, \delta\right)=K_{N, 1} \int_{\mathbb{R}^{N}}\left|\nabla h_{k}\right| d x .
$$

Thus there exists an increasing sequence $n_{k}$ such that for all $n \geq n_{k}$,

$$
I_{1, n}\left(h_{k}\right) \leq K_{N, 1} \int_{\mathbb{R}^{N}}\left|\nabla h_{k}\right| d x+\frac{1}{k} .
$$

Define the sequence $g_{n}$ by $g_{n}=h_{k}$ if $n_{k}<n \leq n_{k+1}$, where $n_{0}=0$. Then for all $n_{k}<n \leq n_{k+1}$,

$$
I_{1, n}\left(g_{n}\right) \leq K_{N, 1} \int_{\mathbb{R}^{N}}\left|\nabla h_{k}\right| d x+\frac{1}{k},
$$


which shows that

$$
\limsup _{n \rightarrow \infty} I_{1, n}\left(g_{n}\right) \leq K_{N, 1} \int_{\mathbb{R}^{N}}|\nabla g| d x .
$$

We now prove the following result which is a partial answer to Questions 3 and 4 . It was announced in [7] for the one-dimensional case.

Theorem 3. Let $\left(g_{n}\right)_{n \in \mathbb{N}}$ be a sequence in $L^{p}\left(\mathbb{R}^{N}\right), 1 \leq p<+\infty$, converging in $L^{p}\left(\mathbb{R}^{N}\right)$ to some $g \in L^{p}\left(\mathbb{R}^{N}\right)$, and $\left(\delta_{n}\right)_{n \in \mathbb{N}}$ be a sequence of positive numbers with $\lim _{n \rightarrow \infty} \delta_{n}=0$. Suppose that

$$
\sup _{n \in \mathbb{N}} \int_{\substack{\mathbb{R}^{N} \times \mathbb{R}^{N} \\\left|g_{n}(x)-g_{n}(y)\right|>\delta_{n}}} \frac{\delta_{n}^{p}}{|x-y|^{N+p}} d x d y<+\infty .
$$

Then $g \in W^{1, p}\left(\mathbb{R}^{N}\right)$ if $p>1$ and $g \in B V\left(\mathbb{R}^{N}\right)$ if $p=1$. Moreover,

$$
\liminf _{n \rightarrow \infty} \iint_{\substack{\mathbb{R}^{N} \times \mathbb{R}^{N} \\\left|g_{n}(x)-g_{n}(y)\right|>\delta_{n}}} \frac{\delta_{n}^{p}}{|x-y|^{N+p}} d x d y \geq c_{N, p} \int_{\mathbb{R}^{N}}|\nabla g|^{p} d x
$$

for some positive constant $c_{N, p}$.

We first prove a technical lemma which plays the same role as [2] Lemma 2] in the proof of [2, Theorem 1]. Its proof is based on that of [2, Lemma 2] and Egorov's theorem (see e.g. [10]).

Lemma 7. Let $\mathcal{I}$ be a bounded interval, $\left(g_{n}\right)_{n \in \mathbb{N}}$ be a sequence in $L^{p}(\mathcal{I}), 1 \leq p<+\infty$, converging in $L^{p}(\mathcal{I})$ to some $g \in L^{p}(\mathcal{I})$, and $\left(\delta_{n}\right)_{n \in \mathbb{N}}$ be a sequence of positive numbers with $\lim _{n \rightarrow \infty} \delta_{n}=0$. Then

$$
\liminf _{n \rightarrow \infty} \iint_{\substack{\mathcal{I} \times \mathcal{I} \\\left|g_{n}(x)-g_{n}(y)\right|>\delta_{n}}} \frac{\delta_{n}^{p}}{|x-y|^{p+1}} d x d y \geq c_{p} \frac{1}{|\mathcal{I}|^{p-1}}|\underset{\mathcal{I}}{\operatorname{ess} \sup } g-\underset{\mathcal{I}}{\operatorname{essinf}} g|^{p},
$$

for some positive constant $c_{p}$ depending only on $p$.

Proof. Without loss of generality suppose that $\mathcal{I}=[0,1]$, ess $\sup _{\mathcal{I}} g=s_{+}, \operatorname{ess}_{\inf _{\mathcal{I}}} g=$ $s_{-}\left(-\infty<s_{-}, s_{+}<+\infty\right)$, and $s_{+}-s_{-}=1$.

Take $0<\delta \ll 1$ small enough to ensure that there are (density) points $t_{+}, t_{-} \in$ $[40 \delta, 1-40 \delta] \subset[0,1]$ with

$$
\left\{\begin{array}{l}
\left|\left[t_{+}-40 \delta, t_{+}+40 \delta\right] \cap\left[g>\frac{4}{5} s_{+}+\frac{4}{5} s_{-}\right]\right|>70 \delta, \\
\left|\left[t_{-}-40 \delta, t_{-}+40 \delta\right] \cap\left[g<\frac{4}{5} s_{-}+\frac{1}{5} s_{+}\right]\right|>70 \delta .
\end{array}\right.
$$


We will assume as well that $g_{n}$ converges to $g$ for almost every $x \in \mathcal{I}$. Thus, by Egorov's theorem (see [10]), there exists a constant $n_{0}$ such that for all $n \geq n_{0}$, we have $\delta_{n} \leq \delta$ and

$$
\left\{\begin{array}{l}
\left|\left[t_{+}-40 \delta, t_{+}+40 \delta\right] \cap\left[g_{n}>\frac{3}{4} s_{+}+\frac{1}{4} s_{-}\right]\right|>60 \delta, \\
\left|\left[t_{-}-40 \delta, t_{-}+40 \delta\right] \cap\left[g_{n}<\frac{3}{4} s_{-}+\frac{1}{4} s_{+}\right]\right|>60 \delta .
\end{array}\right.
$$

Fix $n \geq n_{0}$, take $K \in \mathbb{Z}_{+}$such that $\delta_{n}<2^{-K} \leq 2 \delta_{n}$ and set

$$
J=\left\{j \in \mathbb{Z}_{+} ; \frac{3}{4} s_{-}+\frac{1}{4} s_{+}<j 2^{-K}<\frac{3}{4} s_{+}+\frac{1}{4} s_{-}\right\} .
$$

Then

$$
|J| \geq 2^{K-1}-2 \approx 1 / \delta_{n}
$$

Define (for notational ease)

$$
A_{j}=\left\{x \in[0,1] ;(j-1) 2^{-K} \leq g_{n}(x)<j 2^{-K}\right\}, \quad B_{j}=\bigcup_{j^{\prime}<j} A_{j^{\prime}}, \quad \forall j \in \mathbb{Z} .
$$

Since the sets $A_{j}$ are disjoint, it follows from (4.4) that

$$
\operatorname{card}(G) \geq 2^{K-2}-3 \approx 1 / \delta_{n}
$$

where (for notational ease)

$$
G=\left\{j \in J ;\left|A_{j}\right|<2^{-K+2}\right\} .
$$

For each $j \in G$, set $\lambda_{1, j}=\left|A_{j}\right|$ and consider the function $\psi_{j}(t)$ defined as follows:

$$
\psi_{j}(t)=\left|[t-40 \delta, t+40 \delta] \cap B_{j}\right|, \quad \forall t \in[40 \delta, 1-40 \delta] .
$$

Applying the same method as in [2, Lemma 2], we deduce that there exist $\lambda_{j}>0$ and $t_{j} \in[t-40 \delta, t+40 \delta]$ such that

$$
\left\{\begin{array}{l}
\left|\left[t_{j}-40 \lambda_{j}, t_{j}+40 \lambda_{j}\right] \cap B_{j}\right|=40 \lambda_{j}, \\
\lambda_{j} / 4 \leq\left|\left[t_{j}-40 \lambda_{j}, t_{j}+40 \lambda_{j}\right] \cap A_{j}\right| \leq \lambda_{j} .
\end{array}\right.
$$

It follows that $\lambda_{j} \lesssim \delta_{n}$. The rest of the proof is similar to the one of [2, Lemma 2].

Proof of Theorem 3. The proof is the same as that of [2, Theorem 1], with the use of Lemma 7] in place of [2, Lemma 1]. The details are left to the reader.

Finally, we present a special case where the $\Gamma$-convergence of a sequence of functionals can be established.

Consider the functionals $J_{n}$ and $J$ on $L^{p}\left(\mathbb{R}^{N}\right)$ defined as follows:

$$
J_{n}(g)=\int_{\mathbb{R}^{N}} \int_{\mathbb{R}^{N}} \frac{F_{n}(|g(x)-g(y)|)}{|x-y|^{N+p}} d x d y, \quad \forall n \in \mathbb{N},
$$


and

$$
J(g)=\left\{\begin{aligned}
K_{N, p} & \int_{\mathbb{R}^{N}}|\nabla g|^{p} d x \\
& \text { if } g \in W^{1, p}\left(\mathbb{R}^{N}\right) \text { for } p>1, \text { resp. } g \in B V\left(\mathbb{R}^{N}\right) \text { for } p=1 \\
+\infty \quad & \text { otherwise, }
\end{aligned}\right.
$$

for all $g \in L^{p}\left(\mathbb{R}^{N}\right)$.

We have the following

Theorem 4. Let $1 \leq p<+\infty$. Assume $F_{n}:[0,+\infty) \rightarrow[0,+\infty)$ is convex on the interval $[0,1]$ for all $n \in \mathbb{N}$, and satisfies hypotheses (i)-(iii) of Theorem 1 . Then $\left(J_{n}\right)$ $\Gamma$-converges to $J$ on $L^{p}\left(\mathbb{R}^{N}\right)$.

As a consequence of Theorem 4 , we have

Corollary 3. Let $F_{n}$ be defined as follows:

$$
F_{n}(t)= \begin{cases}\varepsilon_{n} t^{p+\varepsilon_{n}} & \text { if } 0 \leq t \leq 1, \\ \varepsilon_{n} & \text { otherwise, }\end{cases}
$$

for some sequence $\left(\varepsilon_{n}\right)$ converging to 0 . Then $\left(J_{n}\right) \Gamma$-converges to $J$.

In order to prove Theorem 4, one needs the following two lemmas.

Lemma 8. Let $1 \leq p<+\infty$ and $F_{n}:[0,+\infty) \rightarrow[0,+\infty)$ satisfy hypotheses (i)-(iii) of Theorem 1 and $\left(g_{n}\right)_{n \in \mathbb{N}} \subset L^{p}(\mathbb{R}) \cap C_{b}^{2}(\mathbb{R})$. Assume that $g_{n}$ converges to $g$ in $L^{p}(\mathbb{R})$ and $C_{b}^{2}(\mathbb{R})$. Then

$$
\liminf _{n \rightarrow \infty} \int_{\mathbb{R}} \int_{0}^{\infty} \frac{F_{n}\left(\left|g_{n}(x+h)-g_{n}(x)\right|\right)}{h^{p+1}} d h d x \geq \int_{\mathbb{R}}\left|g^{\prime}\right|^{p} d x .
$$

We recall that

$$
C_{b}^{2}\left(\mathbb{R}^{N}\right)=\left\{g \in C^{2}\left(\mathbb{R}^{N}\right) ;\|g\|_{L^{\infty}}+\|D g\|_{L^{\infty}}+\left\|D^{2} g\right\|_{L^{\infty}}<+\infty\right\},
$$

with the norm

$$
\|g\|_{C_{b}^{2}}=\|g\|_{L^{\infty}}+\|D g\|_{L^{\infty}}+\left\|D^{2} g\right\|_{L^{\infty}}, \quad \forall g \in C_{b}^{2}\left(\mathbb{R}^{N}\right) .
$$

Proof. Fix $\varepsilon>0$ arbitrary. Take $\delta>0$ such that

$$
\int_{A_{\delta}}\left|g^{\prime}\right|^{p} d x>(1-\varepsilon) \int_{\mathbb{R}}\left|g^{\prime}\right|^{p} d x
$$

where

$$
A_{\delta}:=\left\{x \in \mathbb{R} ;\left|g^{\prime}(x)\right|>2 \delta\right\} .
$$

Since $g_{n}$ converges to $g$ in $C^{1}(\mathbb{R})$, there exists some $n_{\varepsilon} \in \mathbb{N}$ such that

$$
\left|g_{n}^{\prime}(x)\right|>\delta, \quad \forall x \in A_{\delta} .
$$


Hence since $\left(g_{n}\right)$ is bounded in $C_{b}^{2}(\mathbb{R})$, it follows that

$$
\left|g_{n}(x+h)-g_{n}(x)\right| \geq(1-\varepsilon) h\left|g^{\prime}(x)\right|, \quad \forall x \in A_{\delta}, \forall 0<h<\tau,
$$

for some $\tau>0$ and for all $n \geq n_{\varepsilon}$. Thus since $F_{n}$ is non-decreasing, one gets

$$
\begin{aligned}
\liminf _{n \rightarrow \infty} \int_{\mathbb{R}} \int_{0}^{\infty} \frac{F_{n}\left(\left|g_{n}(x+h)-g_{n}(x)\right|\right)}{h^{p+1}} d h d x \\
\quad \geq \liminf _{n \rightarrow \infty} \int_{A_{\delta}} \int_{0}^{\tau} \frac{F_{n}\left((1-\varepsilon) h\left|g_{n}^{\prime}(x)\right|\right)}{h^{p+1}} d h d x .
\end{aligned}
$$

On the other hand, from (i),

$$
\begin{aligned}
\liminf _{n \rightarrow \infty} \int_{A_{\delta}} \int_{0}^{\tau} \frac{F_{n}\left((1-\varepsilon) h\left|g_{n}^{\prime}(x)\right|\right)}{h^{p+1}} d h d x & \\
& \geq \liminf _{n \rightarrow \infty}(1-\varepsilon)^{p} \int_{A_{\delta}}\left|g_{n}^{\prime}\right|^{p} d x \int_{0}^{\tau \delta / 2} F_{n}(t) t^{-(p+1)} d t .
\end{aligned}
$$

Thus it follows from (ii) and (iii) that

$$
\liminf _{n \rightarrow \infty} \int_{\mathbb{R}} \int_{0}^{\infty} \frac{F_{n}\left(\left|g_{n}(x+h)-g_{n}(x)\right|\right)}{h^{p+1}} d h d x \geq(1-\varepsilon)^{p} \liminf _{n \rightarrow \infty} \int_{A_{\delta}}\left|g_{n}^{\prime}\right|^{p} d x,
$$

which implies, from 4.7 ,

$$
\liminf _{n \rightarrow \infty} \int_{\mathbb{R}} \int_{0}^{\infty} \frac{F_{n}\left(\left|g_{n}(x+h)-g_{n}(x)\right|\right)}{h^{p+1}} d h d x \geq(1-\varepsilon)^{p+1} \int_{\mathbb{R}}\left|g^{\prime}\right|^{p} d x .
$$

Therefore, since $\varepsilon>0$ is arbitrary,

$$
\liminf _{n \rightarrow \infty} \int_{\mathbb{R}} \int_{0}^{\infty} \frac{F_{n}\left(\left|g_{n}(x+h)-g_{n}(x)\right|\right)}{h^{p+1}} d h d x \geq \int_{\mathbb{R}}\left|g^{\prime}\right|^{p} d x .
$$

Lemma 9. Let $1 \leq p<+\infty$ and $F_{n}:[0,+\infty) \rightarrow[0,+\infty)$ satisfy hypotheses (i)-(iii) of Theorem 1 and $g \in C_{c}^{2}\left(\mathbb{R}^{N}\right)$. Then

$$
\lim _{n \rightarrow \infty} \int_{\mathbb{R}^{N}} \int_{\mathbb{R}^{N}} \frac{F_{n}(|g(x)-g(y)|)}{|x-y|^{p+1}} d x d y=K_{N, p} \int_{\mathbb{R}^{N}}|\nabla g|^{p} d x .
$$

Proof. Without loss of generality, one may assume that

$$
\operatorname{supp} g \subset\left\{x \in \mathbb{R}^{N} ;|x| \leq 1\right\} .
$$

Using the change of variables formula one has

$$
\begin{aligned}
\int_{\mathbb{R}^{N}} \int_{\mathbb{R}^{N}} \frac{F_{n}(|g(x)-g(y)|)}{|x-y|^{p+1}} & d x d y \\
= & \int_{\mathbb{S}^{N-1}} \int_{\mathbb{R}^{N}} \int_{0}^{\infty} \frac{F_{n}(|g(x+h \sigma)-g(x)|)}{h^{p+1}} d h d x d \sigma .
\end{aligned}
$$


Thus by Lemma 8 and Fatou's lemma, one gets

$$
\liminf _{n \rightarrow \infty} \int_{\mathbb{R}^{N}} \int_{\mathbb{R}^{N}} \frac{F_{n}(|g(x)-g(y)|)}{|x-y|^{p+1}} d x d y \geq K_{N, p} \int_{\mathbb{R}^{N}}|\nabla g|^{p} d x .
$$

Hence it suffices to prove that

$$
\limsup _{n \rightarrow \infty} \int_{\mathbb{S}^{N-1}} \int_{\mathbb{R}^{N}} \int_{0}^{\infty} \frac{F_{n}(|g(x+h \sigma)-g(x)|)}{h^{p+1}} d h d x d \sigma \leq K_{N, p} \int_{\mathbb{R}^{N}}|\nabla g|^{p} d x .
$$

Fix $\varepsilon>0$ (arbitrary). Define

$$
A_{\varepsilon}:=\left\{(\sigma, x) \in \mathbb{S}^{N-1} \times \mathbb{R}^{N} ;|\nabla g(x) \cdot \sigma|>2 \varepsilon\right\} .
$$

Choose $0<\tau<1$ such that $\tau\|g\|_{C_{b}^{2}}<\varepsilon^{2}$. Then

$$
|g(x+h \sigma)-g(x)|<(1+\varepsilon) h|\nabla g(x) \cdot \sigma|, \quad \forall 0<h<\tau, \forall(\sigma, x) \in A_{\varepsilon} .
$$

Thus it follows from (i) that

$$
\begin{aligned}
\limsup _{n \rightarrow \infty} \int_{A_{\varepsilon}} \int_{0}^{\tau} \frac{F_{n}(|g(x+h \sigma)-g(x)|)}{h^{p+1}} d h d x d \sigma \\
\leq \limsup _{n \rightarrow \infty} \int_{\mathbb{S}^{N-1}} \int_{\mathbb{R}^{N}} \int_{0}^{\tau} \frac{F_{n}(|(1+\varepsilon) \nabla g(x) \cdot \sigma|)}{h^{p+1}} d h d x d \sigma
\end{aligned}
$$

which implies

$$
\limsup _{n \rightarrow \infty} \int_{A_{\varepsilon}} \int_{0}^{\tau} \frac{F_{n}(|g(x+h \sigma)-g(x)|)}{h^{p+1}} d h d x d \sigma \leq(1+\varepsilon)^{p} K_{N, p} \int_{\mathbb{R}^{N}}|\nabla g|^{p} d x .
$$

On the other hand, from the choice of $\tau$,

$$
\int_{A_{\varepsilon}^{c}} \int_{0}^{\tau} \frac{F_{n}(|g(x+h \sigma)-g(x)|)}{h^{p+1}} d h d x \leq \int_{\mathbb{S}^{N-1}} \int_{|x|<2} \int_{0}^{\tau} \frac{F_{n}(3 \varepsilon h)}{h^{p+1}} d h d x \leq C \varepsilon^{p},
$$

where $A_{\varepsilon}^{c}$ denotes the complement of $A_{\varepsilon}$ in $\mathbb{S}^{N-1} \times \mathbb{R}^{N}$. Hereafter in this proof, $C$ denotes a constant independent of $n$. Thus

$$
\begin{array}{r}
\limsup _{n \rightarrow \infty} \int_{\mathbb{S}^{N-1}} \int_{\mathbb{R}^{N}} \int_{0}^{\tau} \frac{F_{n}(|g(x+h \sigma)-g(x)|)}{h^{p+1}} d h d x d \sigma \\
\quad \leq(1+\varepsilon)^{p} K_{N, p} \int_{\mathbb{R}^{N}}|\nabla g|^{p} d x+C \varepsilon^{p} .
\end{array}
$$

On the other hand, it follows from (iii) that

$$
\lim _{n \rightarrow \infty} \int_{\mathbb{S}^{N-1}} \int_{|x| \leq 2} \int_{\tau}^{\infty} \frac{F_{n}(|g(x+h \sigma)-g(x)|)}{h^{p+1}} d h d x d \sigma=0 .
$$


From 4.9, one gets

$$
\begin{aligned}
\int_{\mathbb{S}^{N-1}} \int_{\tau}^{\infty} \int_{|x| \geq 2} \frac{F_{n}(|g(x+h \sigma)-g(x)|)}{h^{p+1}} d h d x d \sigma \\
=\int_{\mathbb{S}^{N-1}} \int_{\tau}^{\infty} \int_{|x| \geq 2} \frac{F_{n}(|g(x+h \sigma)|)}{h^{p+1}} d h d x d \sigma .
\end{aligned}
$$

Also, from 4.9],

$$
\int_{\mathbb{S}^{N-1}} \int_{\tau}^{\infty} \int_{|x| \geq 2} \frac{F_{n}(|g(x+h \sigma)|)}{h^{p+1}} d h d x d \sigma \leq \int_{\mathbb{S}^{N-1}} \int_{\tau}^{\infty} \int_{\mathbb{R}^{N}} \frac{F_{n}(|g(x)|)}{h^{p+1}} d h d x d \sigma .
$$

Thus

$$
\lim _{n \rightarrow \infty} \int_{\mathbb{S}^{N-1}} \int_{\tau}^{\infty} \int_{|x| \geq 2} \frac{F_{n}(|g(x+h \sigma)-g(x)|)}{h^{p+1}} d h d x d \sigma=0 .
$$

Combining 4.11) and 4.12) yields

$$
\lim _{n \rightarrow \infty} \int_{\mathbb{S}^{N-1}} \int_{\mathbb{R}^{N}} \int_{\tau}^{\infty} \frac{F_{n}(|g(x+h \sigma)-g(x)|)}{h^{p+1}} d h d x d \sigma=0 .
$$

Hence it follows from (4.10) and (4.13) that

$$
\begin{aligned}
\limsup _{n \rightarrow \infty} \int_{\mathbb{S}^{N-1}} \int_{\mathbb{R}^{N}} \int_{0}^{\infty} \frac{F_{n}(|g(x+h \sigma)-g(x)|)}{h^{p+1}} d h d x d \sigma \\
\leq(1+\varepsilon)^{p} K_{N, p} \int_{\mathbb{R}^{N}}|\nabla g|^{p} d x+C \varepsilon^{p} .
\end{aligned}
$$

Therefore, since $\varepsilon>0$ is arbitrary, one has

$$
\limsup _{n \rightarrow \infty} \int_{\mathbb{S}^{N-1}} \int_{\mathbb{R}^{N}} \int_{0}^{\infty} \frac{F_{n}(|g(x+h \sigma)-g(x)|)}{h^{p+1}} d h d x d \sigma \leq K_{N, p} \int_{\mathbb{R}^{N}}|\nabla g|^{p} d x .
$$

\section{Proof of Theorem 4}

Step 1: Proof of property (A). By the same method as in the proof of Proposition 5 . property (A) follows from Lemma 9

Step 2: Proof of property (B). We use some ideas of A. Ponce in the proof of [8, Lemma 12.2]. Let $\left(g_{n}\right)$ be a sequence converging to $g$ in $L^{p}\left(\mathbb{R}^{N}\right)$. Let $\left(\rho_{\delta}\right)$ be a sequence of smooth mollifiers. Set

$$
g_{n, k}(x)= \begin{cases}g_{n}(x) & \text { if }\left|g_{n}(x)\right| \leq k, \\ \frac{k g_{n}(x)}{\left|g_{n}(x)\right|} & \text { otherwise, }\end{cases}
$$

for all $k \in \mathbb{N}$, and

$$
g_{n, k, \delta}=g_{n, k} * \rho_{\delta}, \quad \forall n \in \mathbb{N}, \forall k \in \mathbb{N} .
$$


Then, since $F_{n}$ is convex on $[0,1]$, it follows from (i) and (iii) that

$$
\liminf _{n \rightarrow \infty} J_{n}\left(g_{n, k, \delta}\right) \leq \liminf _{n \rightarrow \infty} J_{n}\left(g_{n, k}\right) .
$$

On the other hand, from Fatou's lemma and Lemma 8 , one gets

$$
\liminf _{n \rightarrow \infty} J_{n}\left(g_{n, k, \delta}\right) \geq J\left(h_{k, \delta}\right)
$$

where

$$
h_{k}(x)= \begin{cases}g(x) & \text { if }|g(x)| \leq k \\ \frac{k g(x)}{|g(x)|} & \text { otherwise }\end{cases}
$$

and

$$
h_{k, \delta}=h_{k} * \rho_{\delta}
$$

Thus, since

$$
\liminf _{\delta \rightarrow 0} J\left(h_{k, \delta}\right) \geq J\left(h_{k}\right),
$$

it follows from 4.14 and 4.15 that

$$
\liminf _{n \rightarrow \infty} J_{n}\left(g_{n, k}\right) \geq J\left(h_{k}\right) .
$$

On the other hand, from (i),

$$
J_{n}\left(g_{n, k}\right) \leq J\left(g_{n}\right) .
$$

Therefore,

$$
\liminf _{n \rightarrow \infty} J_{n}\left(g_{n}\right) \geq J\left(h_{k}\right), \quad \forall k \in \mathbb{N} .
$$

This implies

$$
\liminf _{n \rightarrow \infty} J_{n}\left(g_{n}\right) \geq J(g) .
$$

However, without the assumption on the convexity of $\left(F_{n}\right)$ on $[0,1]$ in Theorem 4 , one has

Question 5. Assume that $F_{n}:[0,+\infty) \rightarrow[0,+\infty)$ satisfies hypotheses (i)-(iii) of Theorem 1 , and $J_{n}$ and $J$ are defined by (4.5) and (4.6). Does $\left(J_{n}\right) \Gamma$-converge to $J$ on $L^{p}\left(\mathbb{R}^{N}\right)$ for all $p \geq 1$ ?

Acknowledgments. The author is deeply grateful to Prof. H. Brezis for suggesting the problem and for his encouragement. He would also like to thank warmly Prof. J. Bourgain for the collaboration leading to paper [2]. The methods presented in this paper rely heavily on ideas coming from [2]. 


\section{References}

[1] Bourgain, J., Brezis, H., Mironescu, P.: Another look at Sobolev spaces. In: Optimal Control and Partial Differential Equations, a volume in honour of A. Bensoussan's 60th birthday, J. L. Menaldi, E. Rofman and A. Sulem (eds.), IOS Press, 439-455 (2001) Zbl pre02134074

[2] Bourgain, J., Nguyen, H.-M.: A new characterization of Sobolev spaces. C. R. Math. Acad. Sci. Paris 343, 75-80 (2006) Zbl 1109.46034 MR 2242035

[3] Braides, A.: $\Gamma$-convergence for Beginners. Oxford Lecture Ser. Math. Appl., 22. Oxford Univ. Press, Oxford (2002) Zbl pre01865939 MR 1968440

[4] Brezis, H.: Analyse Fonctionnelle. Théorie et Applications. Mathématiques appliquées pour la maîtrise, Masson (1983) Zbl 0511.46001 MR 0697382

[5] Brezis, H.: How to recognize constant functions. Connections with Sobolev spaces. Uspekhi Mat. Nauk 57, no. 4, 59-74 (2002) (in Russian); English transl.: Russian Math. Surveys 57, 693-708 (2002) Zbl 1072.46020 MR 1942116

[6] Dal Maso, G.: An Introduction to $\Gamma$-convergence. Progr. Nonlinear Differential Equations Appl. 8, Birkhäuser Boston, Boston, MA (1993) Zbl 0816.49001 MR 1201152

[7] Nguyen, H.-M.: Some new characterizations of Sobolev spaces. J. Funct. Anal. 237, 689-720 (2006) Zbl pre05045603 MR 2230356

[8] Ponce, A. C.: A new approach to Sobolev spaces and connections to $\Gamma$-convergence. Calc. Var. Partial Differential Equations 19, 229-255 (2004) MR 2033060

[9] Stein, E.: Singular Integrals and Differentiability Properties of Functions. Princeton Univ. Press (1970) Zbl 0207.13501 MR 0290095

[10] Wheeden, R., Zygmund, A.: Measure and Integral: An Introduction to Real Analysis. Dekker (1977) Zbl 0362.26004 MR 0492146 